\title{
Rapamycin-treated human endothelial cells preferentially activate allogeneic regulatory $\mathrm{T}$ cells
}

\author{
Chen Wang, ${ }^{1}$ Tai Yi, ${ }^{1}$ Lingfeng Qin, ${ }^{2}$ Roberto A. Maldonado, ${ }^{3}$ Ulrich H. von Andrian, ${ }^{3}$ \\ Sanjay Kulkarni, ${ }^{2}$ George Tellides, ${ }^{2}$ and Jordan S. Pober ${ }^{1}$ \\ 1Department of Immunobiology and 2Department of Surgery, Yale University School of Medicine, New Haven, Connecticut, USA. \\ ${ }^{3}$ Immune Disease Institute, Harvard Medical School, Boston, Massachusetts, USA.
}

\begin{abstract}
Human graft endothelial cells (ECs) can act as antigen-presenting cells to initiate allograft rejection by host memory T cells. Rapamycin, an mTOR inhibitor used clinically to suppress $\mathrm{T}$ cell responses, also acts on DCs, rendering them tolerogenic. Here, we report the effects of rapamycin on EC alloimmunogenicity. Compared with mock-treated cells, rapamycin-pretreated human ECs (rapa-ECs) stimulated less proliferation and cytokine secretion from allogeneic $\mathrm{CD}^{+}$memory cells, an effect mimicked by shRNA knockdown of mTOR or raptor in ECs. The effects of rapamycin persisted for several days and were linked to upregulation of the inhibitory molecules PD-L1 and PD-L2 on rapa-ECs. Additionally, rapa-ECs produced lower levels of the inflammatory cytokine IL-6. $\mathrm{CD}^{+}$memory cells activated by allogeneic rapa-ECs became hyporesponsive to restimulation in an alloantigen-specific manner and contained higher percentages of suppressive $\mathrm{CD} 4^{+} \mathrm{CD} 25^{\mathrm{hi}} \mathrm{CD} 127^{\mathrm{lo}} \mathrm{FoxP3}{ }^{+}$ cells that did not produce effector cytokines. In a human-mouse chimeric model of allograft rejection, rapamycin pretreatment of human arterial allografts increased graft EC expression of PD-L1 and PD-L2 and reduced subsequent infiltration of allogeneic effector $T$ cells into the artery intima and intimal expansion. Preoperative conditioning of allograft ECs with rapamycin could potentially reduce immune-mediated rejection.
\end{abstract}

\section{Introduction}

Immune-mediated rejection represents a significant barrier to the success of solid organ transplantation. Host alloreactive CD4 ${ }^{+}$ $\mathrm{T}$ cells that cross-react to recognize non-self (allogeneic) MHC molecules expressed on donor-derived APCs carried along in the graft are critical mediators of rejection (1). APC function requires expression of MHC-peptide complexes (the antigen or signal 1), expression of antigen-independent costimulators (signal 2), and, often, production of activating cytokines (signal 3). Human vascular ECs in situ basally express MHC class I and class II molecules $(2,3)$; costimulatory molecules such as LFA-3 (CD58), ICOS ligand (CD275), OX40 ligand (CD252), 41BB ligand (CD137L), CD40, and GITR ligand (4); and cytokines such as IL-1 $\alpha$, IL-6, IL-15, and IL-18 (5-8) that can contribute to T cell activation and differentiation. This panoply of molecular signals enables human ECs to function as APCs and activate allogeneic memory, but not naive, $\mathrm{CD}^{+} \mathrm{T}$ cells to proliferate, secrete effector cytokines, and reject human allografts in immunodeficient mouse hosts (4, 9-11). Human ECs can also deliver inhibitory signals via expression of PD-L1 (also known as B7-H1 or CD274) and PD-L2 (also known as B7-DC or CD273), which bind to PD-1 (CD279) on T cells (12, 13). The balance of positive and negative signals on ECs affects the net outcome of memory $\mathrm{T}$ cell responses.

Human peripheral blood $\mathrm{CD}^{+} \mathrm{T}$ cells in adults are approximately equally divided between naive and memory cells. Memory $\mathrm{T}$ cells are important mediators of rejection in clinical transplantation $(14,15)$, and a significant percentage of circulating memory cells in human transplant recipients, likely generated during prior microbial infections, cross-react to recognize donor graft allo-

Conflict of interest: The authors have declared that no conflict of interest exists. Citation for this article: J Clin Invest. 2013;123(4):1677-1693. doi:10.1172/JCI66204. antigens (16). Furthermore, the frequency of donor-specific memory $\mathrm{T}$ cells correlates with the likelihood of rejection (17). Because alloreactive memory $\mathrm{T}$ cells can be activated by ECs, it is believed that allograft ECs are sufficient to trigger rejection by directly presenting alloantigen to and activating host alloreactive memory T cells (18) in the absence of graft-derived professional APCs. This is in marked contrast to the phenotype in rats, in which the passenger leukocytes, defined as professional APCs present within a solid organ allograft, have been shown to be necessary to initiate rejection (19). Despite this pathogenic role, there are no clinical therapies aimed at reducing EC alloimmunogenicity.

$\mathrm{CD}^{+}$effector $\mathrm{T}$ cells are a heterogeneous population that can be divided into multiple subsets defined by their cytokine profiles. Among these subsets are Th1 cells, which express the master transcription factor T-bet and secrete IFN- $\gamma$; Th2 cells, which express the master transcription factor GATA3 and secrete IL-4, IL-5, and IL-13; and Th17 cells, which express the master transcription factor ROR $\gamma$ T and secrete IL-17A and IL-17F (20, 21). All three subsets are capable of causing allograft destruction (22-25). A fourth subset of $\mathrm{CD} 4^{+} \mathrm{T}$ cells, called Tregs, can suppress immune responses and comprises at least two major populations: natural and inducible Tregs (26). Natural Tregs develop in the thymus, recognize self antigens, control autoimmunity, and express high levels of CD25 and the transcription factor FoxP3. Although activated human $\mathrm{T}$ effector cells also express CD25 and FoxP3, expression in natural Tregs is generally higher and more sustained, a difference attributable to reduced methylation of DNA in a critical transcriptional control region of the FOXP3 gene known as the Treg-specific demethylated region (TSDR) (27). Inducible Tregs (iTregs) form in the periphery and develop or convert from conventional CD25-negative $\mathrm{CD} 4^{+} \mathrm{T}$ cells that may also have the potential to become effector T cells (26). Such iTregs, which may 
or may not stably express FoxP3, are specific for non-self antigens (including alloantigens) and likely restrain immune responses to environmental antigens (28-30). Both natural and inducible Tregs prevent rejection and induce allograft tolerance in several models of transplantation (31-33).

The milieu in which a $\mathrm{CD}^{+} \mathrm{T}$ cell becomes activated largely determines the acquisition of specific effector or regulatory functions (reviewed in refs. 20,21). In the case of Tregs, development of some types of iTregs requires TGF- $\beta$ and the absence of IL- 6 (26), whereas other types are induced by IL-10 (34). In addition to cytokines, formation of iTregs can also be driven by cell surface signals provided by APCs (35), such as PD-L1, which promotes iTreg differentiation even in the absence of TGF- $\beta$ (36). Although these observations are based on studies of naive $\mathrm{T}$ cell commitment, human memory $\mathrm{CD}^{+} \mathrm{T}$ cells display plasticity and are capable of acquiring regulatory activity following antigenic restimulation (5, 37-39). The relationship between human ECs and Tregs is incompletely understood, and it is unknown whether human ECs can be induced to express molecules that favor activation or differentiation of tolerance-promoting Tregs instead of pathogenic inflammatory effectors $\mathrm{T}$ cells.

Since its approval in 1999, rapamycin has been used as an immunosuppressant in human transplant recipients (40). Rapamycin acts by forming a complex with a cytosolic prolyl isomerase known as FK-BP12, and the drug-protein complex acts as an inhibitor of mTOR, a $289-\mathrm{kDa}$ serine/threonine protein kinase that integrates environmental cues to regulate cell growth and division (41). More specifically, rapamycin targets mTOR as part of an mTOR signaling complex (called mTORC1) that also contains a protein called raptor that is critical for its function. Prolonged rapamycin treatment has been shown to inhibit another mTORcontaining signaling complex, known as mTORC2 (42), that lacks raptor but instead contains a protein called rictor that is critical for its function. In CD4 ${ }^{+} \mathrm{T}$ cells, $\mathrm{mTOR}$ is activated in response to IL-2 or other growth-promoting cytokines, and it is believed that rapamycin achieves immune suppression by inhibiting growth factor-driven lymphocyte proliferation. In addition to its effects on $\mathrm{T}$ cells, recent data suggest that rapamycin may also target $\mathrm{mTOR}$ in APCs (43), altering their function. Human and rodent bone marrow-derived DCs treated with rapamycin display decreased expression of MHC and costimulatory molecules, an effect resistant to maturation signals. These rapamycin-treated DCs poorly stimulate allogeneic $\mathrm{T}$ cells, induce alloantigen-specific anergy, and selectively expand FoxP3 ${ }^{+}$regulatory $\mathrm{T}$ cells (44-46). In rodent models of allograft rejection, infusion of rapamycin-treated, alloantigen-pulsed DCs into the host prior to transplant results in prolonged graft survival, associated with increased frequencies of FoxP $^{+}$cells in the spleen and infiltrating into the graft $(45,47)$. Based on these data, we hypothesized that rapamycin may also have tolerance-promoting effects on human ECs.

Here we show that pretreatment of cultured human umbilical vein ECs with rapamycin reduces proliferation and cytokine secretion of cocultured allogeneic memory $\mathrm{CD}^{+} \mathrm{T}$ cells, a phenotypic change that can be replicated by shRNA knockdown of mTOR or raptor in the ECs. Rapamycin treatment or knockdown of mTOR or raptor affected both costimulator molecule expression and cytokine production, but Ab-blocking experiments linked reduced allostimulatory capacity to the upregulation of the inhibitory molecules PD-L1 and PD-L2 on rapa-ECs. T cells activated by rapa-ECs became hyporesponsive to restimulation, in an alloantigen-specific manner, and contained an increased ratio of $\mathrm{CD} 4^{+} \mathrm{CD} 25^{\mathrm{hi}} \mathrm{CD} 127^{\mathrm{lo}}$ $\mathrm{FoxP}^{+}$to $\mathrm{CD}^{+}{ }^{+} \mathrm{CD} 25^{+} \mathrm{FoxP}^{-} \mathrm{T}$ cells. These FoxP3 ${ }^{+}$cells were functionally suppressive, displayed demethylation of the TSDR within the FOXP3 gene, and did not secrete effector cytokines. Finally, using a humanized mouse model of artery allograft rejection, we demonstrate that treatment of human vessels with rapamycin prior to transplant reduced both the infiltration of allogeneic $T$ cells into the vessel intima and intimal expansion, an effect that coincided with increased expression of PD-L1 and PD-L2 on treated graft ECs and was diminished by PD-1 blockade. These data show that rapamycin reduces alloimmunogenicity and confers tolerance-promoting effects on ECs in vitro and in vivo.

\section{Results}

Rapamycin-pretreated ECs poorly stimulate allogeneic memory $C D 4^{+}$ $T$ cells. To study the effects of rapamycin on EC alloimmunogenicity, we treated MHC class $\mathrm{II}^{+}$ECs with varying doses of rapamycin (up to $100 \mathrm{ng} / \mathrm{ml}$ ) for up to 72 hours, then washed away drug and cocultured pretreated ECs with purified allogeneic resting memory $\mathrm{CD}^{+} \mathrm{T}$ cells. There was no difference in the ability of $\mathrm{T}$ cells to recognize allogeneic MHC class II molecules on control ECs and rapa-ECs, as evidenced by a similar degree of induction of CD25, an activation marker expressed in response to TCR signaling, at 72 hours (Figure 1A). However, T cells stimulated by rapa-ECs secreted less IL- 2 and IFN- $\gamma$ at 24 hours (Figure 1B) and were poorly proliferative compared with $T$ cells cocultured with control EC, an effect dependent on both drug dose and treatment duration (Figure 1C). To determine the longevity of this effect, ECs were treated with rapamycin for 72 hours, washed, cultured in the absence of drug for up to 7 days, and then cocultured with allogeneic memory $\mathrm{CD} 4^{+} \mathrm{T}$ cells. We found that at low doses, rapa-ECs were poorly stimulatory compared with untreated ECs even after 4 days following drug washout, while at high doses, the effects of rapamycin treatment persisted for as long as 7 days (Figure 1D). To determine whether changes in cell surface or soluble factors were responsible for the decreased allostimulatory capacity of rapa-ECs, we cultured control ECs or rapa-ECs across a Transwell from cocultures of untreated autologous ECs and allogeneic memory $\mathrm{CD}^{+} \mathrm{T}$ cells. $\mathrm{T}$ cell proliferation, in the lower chamber, was unaffected when either control ECs or rapa-ECs (Figure 1E) were plated in the upper chamber, suggesting that rapamycin likely reduces EC alloimmunogenicity by altering expression of cell surface molecules.

Because rapamycin is known to directly inhibit effector $\mathrm{CD}^{+}$ $\mathrm{T}$ cells, it is possible that the observed inhibition of $\mathrm{T}$ cell alloresponses resulted from carryover of rapamycin from the pretreatment period into the EC-T cell cocultures, where it directly acts on $\mathrm{T}$ cells. To address this possibility and to confirm that the effect of rapamycin was mediated by inhibition of $\mathrm{mTOR}$ in the ECs, we used shRNA to knock down mTOR in the ECs (Figure 2A). Since carryover of shRNA is extremely unlikely, this approach allowed us to distinguish the effects of specifically inhibiting mTOR in ECs. Similar to rapa-ECs, mTOR-knockdown ECs poorly stimulated proliferation of allogeneic memory $\mathrm{CD}^{+} \mathrm{T}$ cells (Figure $2 \mathrm{~B}$ ), suggesting that rapamycin reduces the allostimulatory capacity of ECs by inhibiting mTOR signaling. To determine which specific mTOR signaling complex regulates EC alloimmunogenicity, we knocked down essential components of each complex, raptor for mTORC1 and rictor for mTORC2 (Figure 2C). These experiments revealed that allogeneic 
A
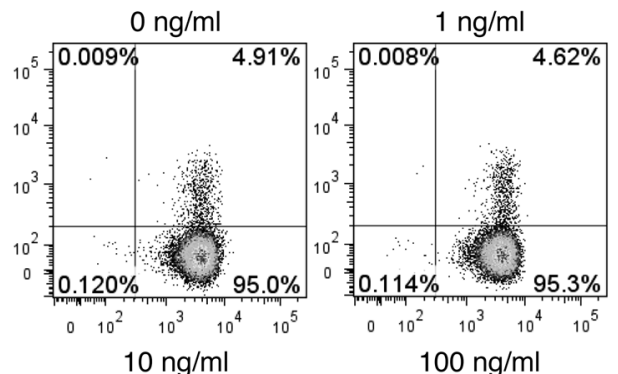

Freshly isolated
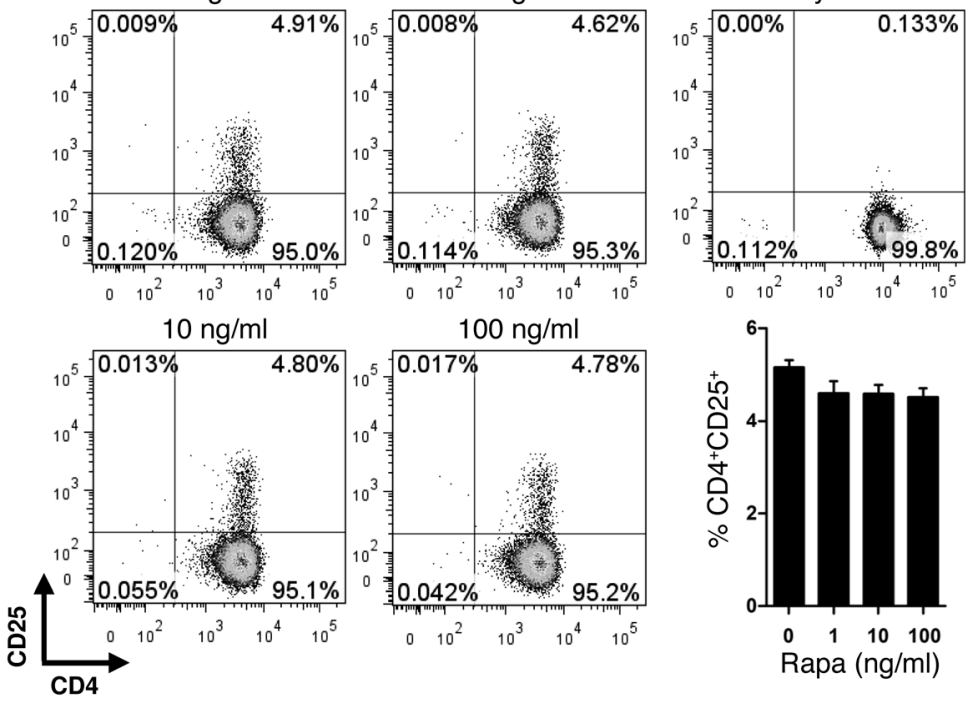

B

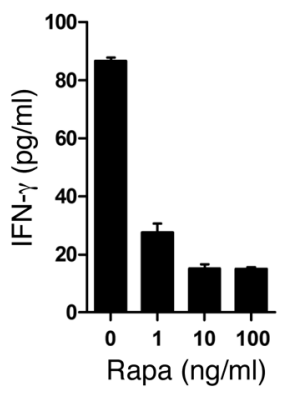

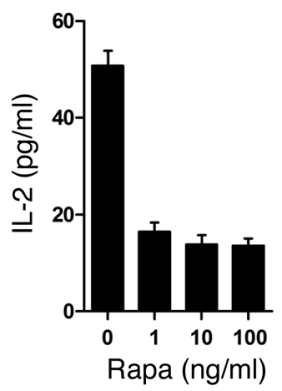

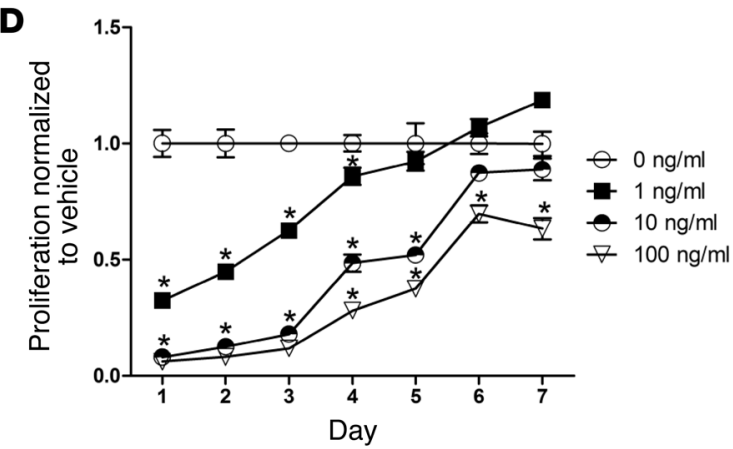

$\mathbf{E}$

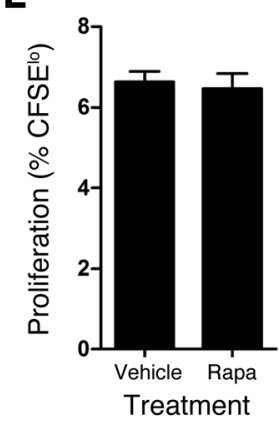

Figure 1

Rapa-ECs poorly stimulate allogeneic memory CD4+ $\mathrm{T}$ cells. (A) CD4+ memory cells were cocultured with allogeneic control ECs or rapa-ECs for 72 hours and analyzed by FACS. Freshly isolated T cells were stained and analyzed immediately following isolation. Representative FACS plots are gated on total CD4+ cells. Similar results were seen in 3 independent experiments. (B) Production of IL-2 and IFN- $\gamma$ by CD4+ memory cells cocultured with allogeneic control ECs or rapa-ECs at 24 hours was assessed by ELISA. Similar results were seen in 3 independent experiments. (C) ECs were treated with the indicated doses of rapamycin (rapa) for 24 or 72 hours and cocultured with CFSE-labeled allogeneic CD4+ memory T cells. Proliferation of T cells was assessed after 7 days via CFSE dilution. (D) ECs were treated with the indicated doses of rapamycin for 72 hours, washed, and maintained up to 7 days (in the absence of drug), then cocultured with CFSE-labeled allogeneic CD4+ memory $T$ cells. $T$ cell proliferation was assessed after 7 days and normalized to vehicle control $(0 \mathrm{ng} / \mathrm{ml}$ rapa). (E) Control ECs or rapa-ECs were cultured in a Transwell across from cocultures of untreated ECs and CFSE-labeled allogeneic CD4+ memory T cells. Proliferation of CFSE-labeled T cells was assessed after 7 days. Mean \pm SEM of triplicate samples is shown from $3(\mathbf{C})$ or $2(\mathbf{D}$ and $\mathbf{E})$ independent experiments. ${ }^{*} P<0.05$.

T cells proliferated less when stimulated by either mTOR- or raptorknockdown ECs, but not when rictor was knocked down (Figure 2D). Interestingly, knockdown of rictor in ECs slightly increased proliferation of cocultured T cells. We conclude that disruption of mTORC1 signaling reduces EC alloimmunogenicity.

Allogeneic memory $C D 4^{+} T$ cells stimulated by rapa-ECs are byporesponsive to restimulation. To distinguish between immunologic ignorance and induction of tolerance, we investigated whether $T$ cells were functionally altered by coculture with rapa-ECs by testing their ability to respond to restimulation. $\mathrm{CD} 4^{+}$memory $\mathrm{T}$ cells first cocultured with allogeneic rapa-ECs, for 72 hours, proliferated less when restimulated by untreated ECs from either the same donor as used in the primary stimulation or third-party donors (Figure $3 \mathrm{~A})$; however, this hyporesponsiveness was more profound when matched ECs were used as restimulators. This suggests that $\mathrm{T}$ cells cocultured with rapa-ECs become hyporesponsive in at least a partly alloantigen-specific manner. It is important to note that after 72 hours of primary stimulation, the percentages of $\mathrm{T}$ cells activated by control and rapa-EC (as judged by CD25+) were equal (Figure 1A). Thus, the decreased proliferation of $\mathrm{T}$ cells cocultured with rapaECs upon restimulation does not reflect differences in the degree of expansion during primary stimulation. The lesser reduced response to third-party ECs could imply some contribution of MHC-independent hyporesponsiveness, but since the ECs were not HLA typed, we cannot rule out the possibility of shared MHC alleles. In either case, the functional alteration was highly dependent on TCR recognition of MHC class II molecules on rapa-ECs, as addition of blocking antibodies to HLA-DR in primary EC-T cell cocultures largely, but not completely, prevented the development of hyporesponsiveness by rapa-EC-primed $\mathrm{T}$ cells during restimulation (Figure $3 \mathrm{~B}$ ). 
A

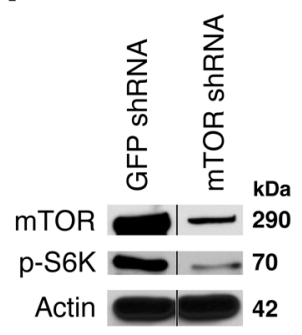

B
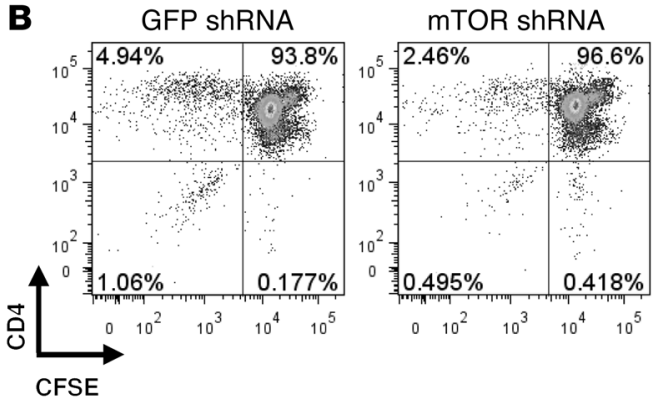

D

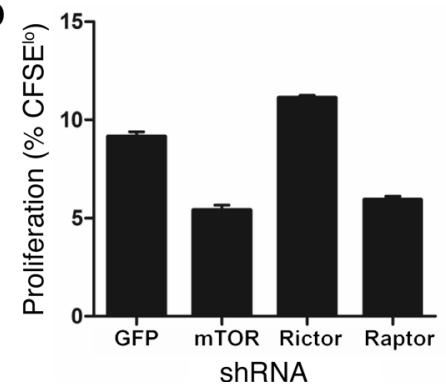

shRNA

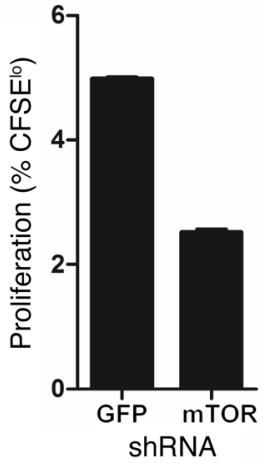

C

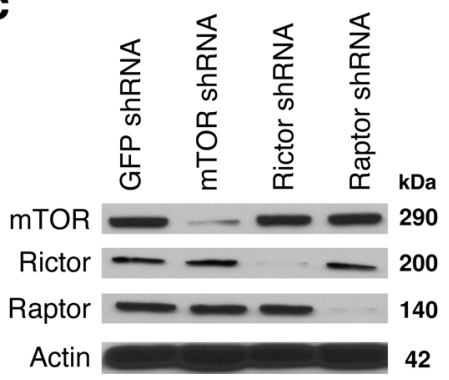

Figure 2

mTOR and raptor knockdown decreases EC alloimmunogenicity. (A) ECs were transduced with shRNA targeting GFP (control) or mTOR as described in Methods. Knockdown was confirmed by Western blot analysis (lanes were run on the same gel, but were not contiguous; breaks are indicated by black vertical line). (B) GFP or mTOR knockdown ECs were cocultured with CFSE-labeled allogeneic CD4+ memory T cells. Proliferation was assessed after 7 days via CFSE dilution. Representative FACS plots are gated on total CD4+ cells. Similar results were seen in 3 independent experiments. (C) ECs were transduced with shRNA targeting GFP (control), mTOR, raptor, or rictor. Knockdown was confirmed by Western blot analysis. (D) Knockdown ECs from C were cocultured with CFSE-labeled allogeneic CD4+ memory T cells. T cell proliferation was assessed after 7 days. Similar results were seen in 2 independent experiments. Mean \pm SEM is shown for representative experiments in $\mathbf{B}$ and $\mathbf{D}$.

This incomplete blockade also may be interpreted as an MHC-independent contribution, but even though HLA-DR is the dominant determinant of direct $\mathrm{CD}^{+} \mathrm{T}$ cell allorecognition, we cannot rule out small contributions from recognition of HLA-DP and HLA-DQ that at not blocked by our antibody.

Rapa-ECs induce an increased percentage of $C D 4^{+} C D 25^{\text {hi }} \mathrm{CD} 127^{l o}$ Fox $\mathrm{P3}^{+}$cells. The observation that allogeneic $\mathrm{CD} 4^{+} \mathrm{T}$ cells stimulated by rapa-ECs become hyporesponsive to restimulation could be due to clonal anergy, to clonal deletion, or to activation or induction of Tregs. We did not detect any differences in cell death (assessed by Annexin V or propidium iodide) in T cells stimulated by control ECs or rapa-ECs (data not shown), arguing against clonal deletion. To address the possibilities of anergy or Treg induction, we cocultured memory CD4 $\mathrm{T}^{+}$cells with allogeneic control ECs or rapa-ECs for 72 hours and FACS sorted activated T cells on the basis of $\mathrm{CD} 4{ }^{+} \mathrm{CD} 25^{+}$expression. Using qRT-PCR, we
A

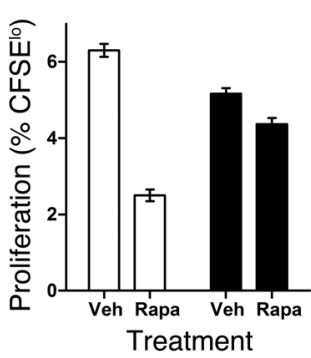

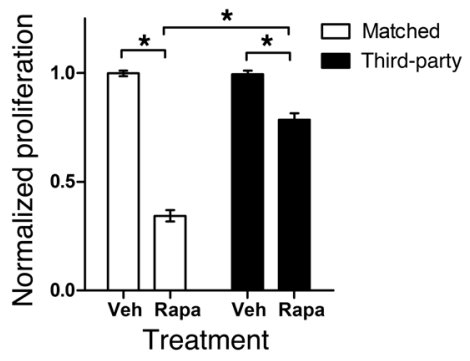

B

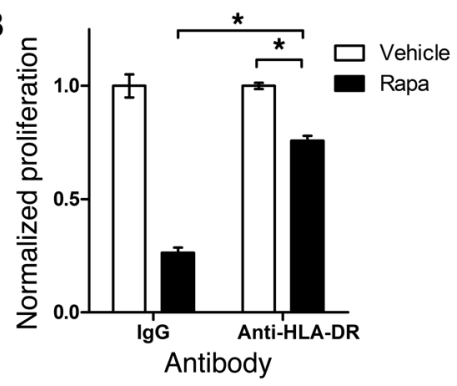

Figure 3

Allogeneic CD4+ memory cells stimulated by rapa-ECs become hyporesponsive to restimulation. (A) Allogeneic CD4+ memory T cells stimulated by control ECs or rapa-ECs for 72 hours (at which time the percentage of activated CD25+ $\mathrm{T}$ cells in each group is equal) were rested, CFSE labeled, and restimulated by fresh ECs from the same donor as used in primary stimulation or from third-party donors. Proliferation of restimulated T cells was assessed by CFSE dilution; percent proliferation (\%CFSE $\left.{ }^{\circ}\right)$ is shown on left, and proliferation normalized to vehicle control is shown on right. (B) Restimulation experiment, as described above, except $30 \mu \mathrm{g} / \mathrm{ml}$ of neutralizing HLA-DR or isotype control antibodies was added to primary cocultures, and T cells were only restimulated by matched ECs. Mean \pm SEM of triplicate samples is shown from 3 (A, normalized data) or 2 (B) independent experiments. ${ }^{*} P<0.05$. 
A
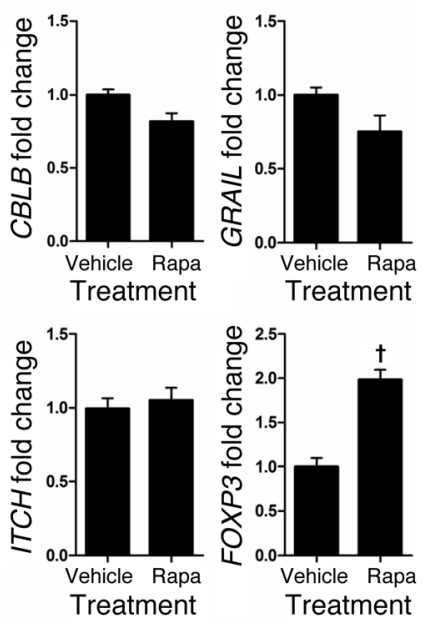

B

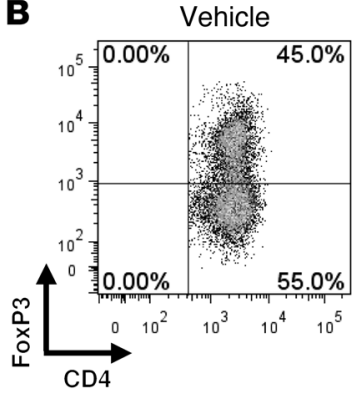

C

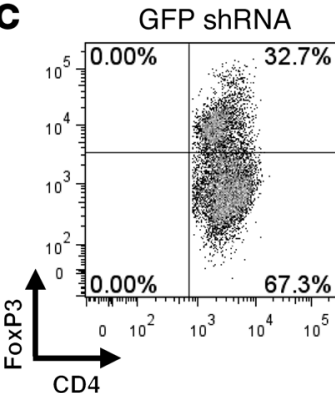

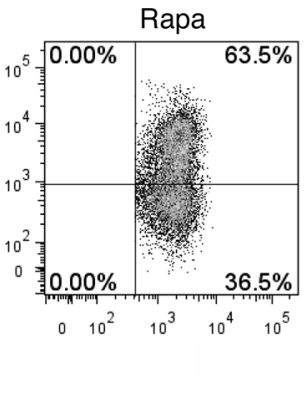

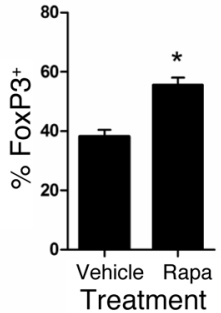

MTOR ShRNA
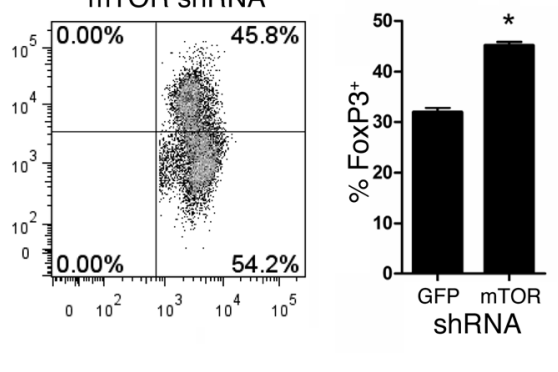

Freshly isolated

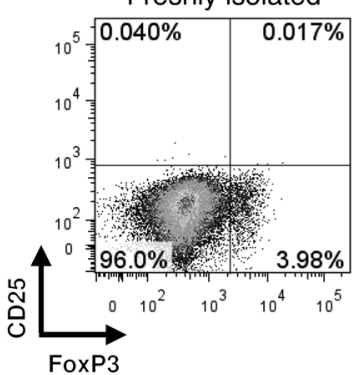

D

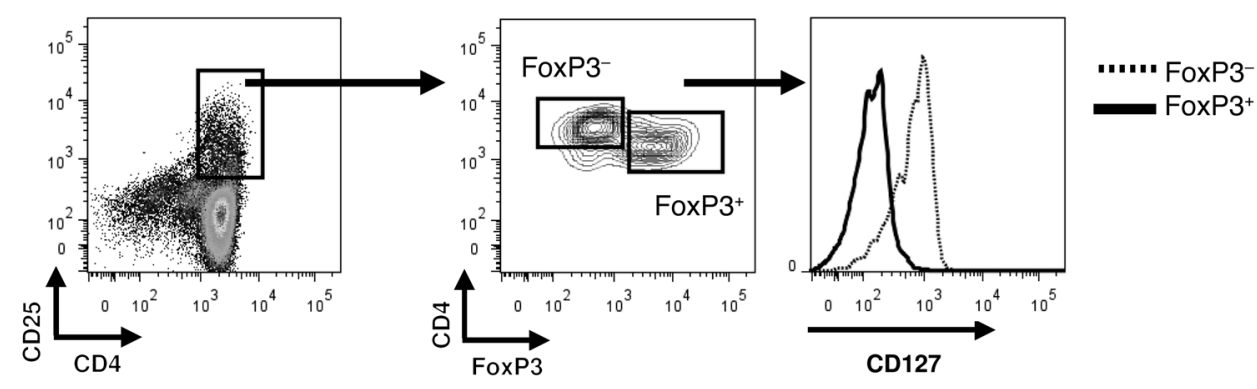

E

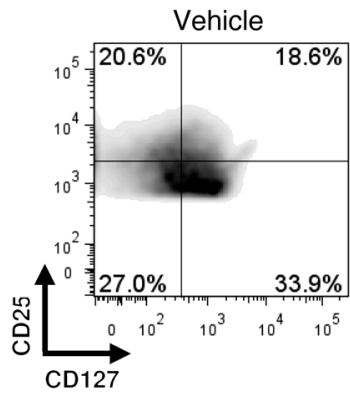

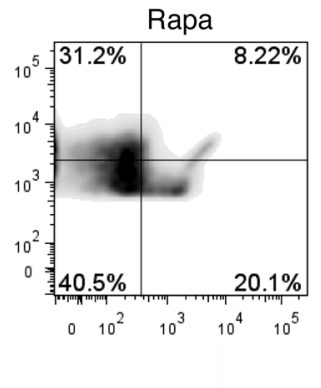

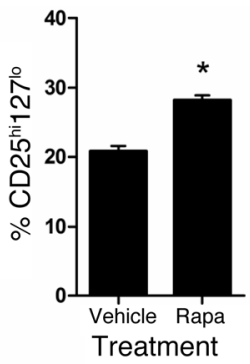

Figure 4

Rapa-ECs increase the percentage of CD4 ${ }^{+} \mathrm{CD} 25^{\text {hi }} \mathrm{CD} 127^{\mathrm{l}} \mathrm{FoxP} 3^{+}$cells among CD25 $5^{+}$activated T cells. (A) Activated T cells cocultured with control ECs or rapa-ECs were FACS sorted on the basis of CD25+ expression, and used for qRT-PCR analysis. All transcripts were normalized to CD3, and expression was then normalized to vehicle control. (B) Allogeneic CD4+ memory T cells were cocultured with control ECs or rapaECs for 7 days and analyzed by intracellular FACS. Freshly isolated T cells were stained and analyzed immediately following isolation. Representative FACS plots are gated on CD4+CD25+ T cells, except for freshly isolated sample, which is gated on total CD4+ cells. (C) CD4+ memory T cells cocultured with allogeneic GFP or mTOR knockdown ECs were analyzed by intracellular FACS. Representative FACS plots are gated on CD4 ${ }^{+}$CD25 ${ }^{+}$T cells. (D) CD127 expression was assessed on FoxP3 ${ }^{+}$and FoxP3- populations in CD25+ ${ }^{+}$cells activated by rapa-ECs. Similar results were seen in 2 additional independent experiments. (E) Cocultures were performed as described in $\mathbf{B}$. Representative plots are gated on CD4 ${ }^{+}$CD25 ${ }^{+}$T cells. Mean \pm SEM of triplicate samples is shown from $2(\mathbf{A}$ and $\mathbf{C})$ or $3(\mathbf{B}$ and $\mathbf{E})$ independent experiments. ${ }^{\dagger} P<0.01,{ }^{*} P<0.05$.

found that $T$ cells activated by rapa-ECs did not express increased transcript levels of the anergy-associated genes GRAIL, ITCH, and $C B L B$ but did contain significantly higher mRNA levels of the Treg master transcription factor FOXP3 (Figure 4A). We were intrigued by this result, given the observation that rapamycin-treated DCs favor activation and expansion of Tregs. To determine whether rapa-ECs have a similar capability, we performed intracellular FACS analysis on allogeneic memory $\mathrm{CD} 4^{+} \mathrm{CD} 25^{-} \mathrm{T}$ cells cocultured with rapa-ECs and found that activated (now $\mathrm{CD} 25^{+}$) T cells, which constituted about $5 \%-10 \%$ of the total $\mathrm{CD}^{+}$popu- 

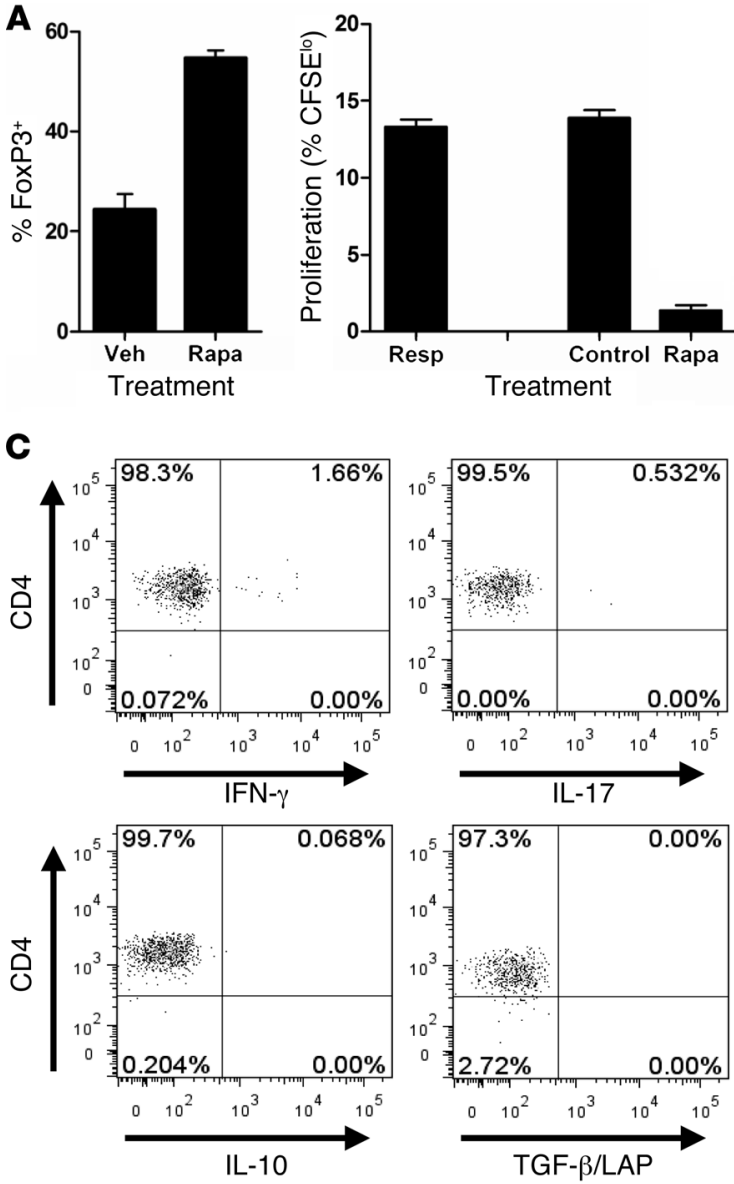

B

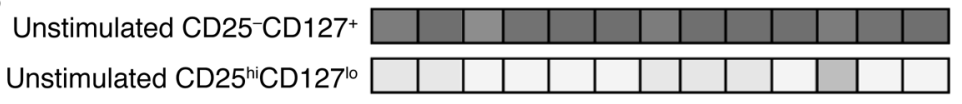

Rapa-EC CD25-CD127+

Rapa-EC CD25 $5^{\text {hCD } 127}$

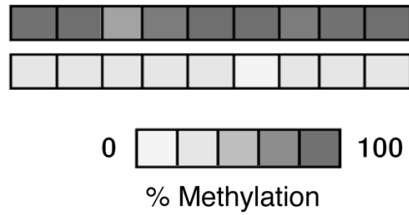

D
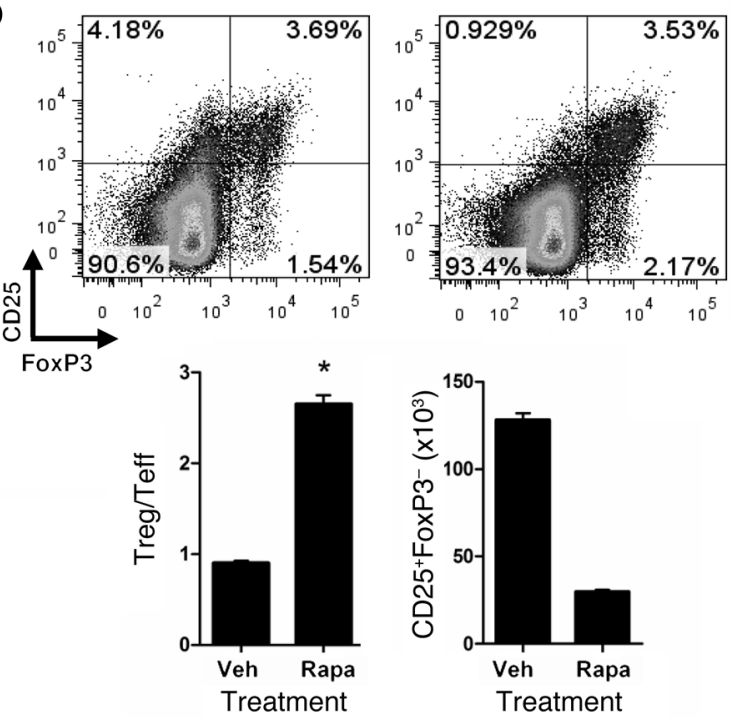

E

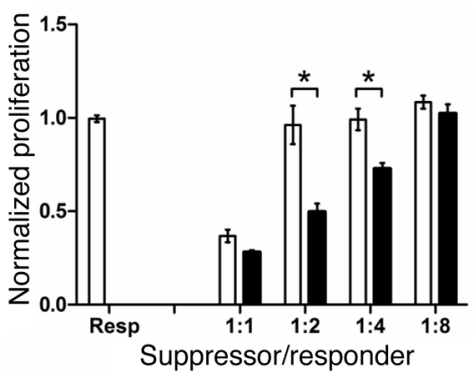

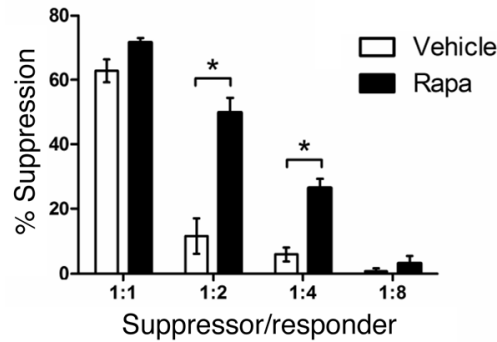

Figure 5

$\mathrm{CD} 4^{+} \mathrm{CD} 25^{\text {hi }} \mathrm{CD} 127^{\mathrm{l}} \mathrm{FoxP} 3^{+}$cells activated by rapa-ECs are functional Tregs. (A) Left: Percentage of FoxP3 $3^{+}$cells in $\mathrm{CD} 25^{+}$pool activated by anti-CD3-loaded $\mathrm{CD}_{2} 2^{+}$control ECs and rapa-ECs. Right: FACS-sorted CD25 hi T cells activated by anti-CD3-loaded CD32+ rapa-ECs were added to fresh cocultures of untreated CD32-transduced ECs and CFSE- labeled responder CD4+ memory T cells at 1:1 ratios. In control wells, equal numbers of freshly isolated T cells were added in place of rapa-EC-primed T cells. Similar results were seen in 3 independent experiments. (B) $C D 4{ }^{+} C D 25^{\text {hi }} C D 127^{10} \mathrm{~T}$ cells activated by rapa-ECs were FACS sorted and used for DNA methylation analysis. Data are representative of 2 independent experiments. (C) CD4+ memory T cells cocultured with allogeneic rapa-ECs for 6 days were stimulated with PMA/ionomycin in the

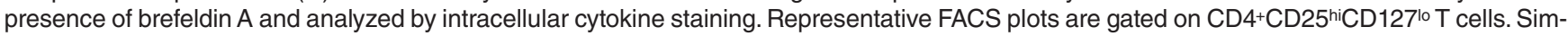
ilar results were seen in 2 independent experiments. (D) Representative FACS plots (gated on CD4+ T cells) of CD4+ memory T cells cocultured with control ECs or rapa-ECs. Treg/effector T (Teff) ratio calculated as $\left(\% \mathrm{CD} 25^{+} \mathrm{FoxP} 3^{+}\right) /\left(\% \mathrm{CD} 25^{+} \mathrm{FoxP} 3^{-}\right)$. Absolute numbers of CD25+FoxP3cells calculated by multiplying the percentage of $\mathrm{CD}_{25}{ }^{+} \mathrm{FoxP} 3^{-}$cells in the $\mathrm{CD}^{+}$gate by the number of $\mathrm{CD}^{+}{ }^{+} \mathrm{T}$ cells used in each coculture. (E) FACS-sorted total CD25+T cells, activated as in $\mathbf{A}$, were titered at varying ratios into fresh cocultures of untreated CD32-transduced ECs and CFSE-labeled responder T cells. Mean \pm SEM of triplicate samples is shown from $3(D)$ or $2(E)$ independent experiments. ${ }^{\star} P<0.05$.

lation in various experiments, contained a greater proportion of FoxP3 $3^{+} \mathrm{T}$ cells compared with cocultures with control ECs (Figure $4 \mathrm{~B})$. These $\mathrm{CD} 25^{+} \mathrm{FoxP}^{+} \mathrm{T}$ cells were CD127 low/negative (Figure $4 \mathrm{D})$. Overall, $\mathrm{CD} 25^{+} \mathrm{T}$ cells activated by rapa-ECs contained a greater percentage of $\mathrm{CD} 25^{\text {hi }} \mathrm{CD} 127^{\text {lo }} \mathrm{T}$ cells (Figure $4 \mathrm{E}$ ) compared with $\mathrm{T}$ cells activated by control ECs; in both groups these CD25 ${ }^{\text {hi }} \mathrm{CD} 127^{\text {lo }}$ cells were greater than $95 \% \mathrm{FoxP}^{+}$(data not shown). It is important to note that in our system, the memory 


\section{Table 1}

Flow cytometric analysis of EC surface costimulatory molecules on control and Rapa-ECs

$\begin{array}{lcccc} & \text { Vehicle (MFI) } & \text { Rapa (MFI) } & \text { IFN- } \gamma(\text { MFI) } & \text { IFN }-\gamma+\text { Rapa (MFI) } \\ \text { HLA-ABC } & 3,104( \pm 71) & 2,479( \pm 312) & 44,399( \pm 550) & 47,999( \pm 1670) \\ \text { HLA-DR } & 0 & 0 & 3,040( \pm 110) & 3,324( \pm 140) \\ \text { LFA-3 } & 2,312( \pm 40) & 2,422( \pm 28) & 2,090( \pm 46) & 2,273( \pm 55) \\ \text { CD40 } & 1,416( \pm 69) & 752( \pm 6)^{\mathrm{A}} & 5,858( \pm 44) & 4,472( \pm 69)^{\mathrm{A}} \\ \text { OX40 ligand } & 74( \pm 7) & 243( \pm 9)^{\mathrm{A}} & 148( \pm 5) & 236( \pm 7)^{\mathrm{A}} \\ \text { GITR ligand } & 75( \pm 1) & 281( \pm 7)^{\mathrm{A}} & 209( \pm 5) & 206( \pm 3) \\ \text { PD-L1 } & 97( \pm 4) & 394( \pm 4)^{\mathrm{A}} & 729( \pm 28) & 1,703( \pm 112)^{\mathrm{A}} \\ \text { PD-L2 } & 91( \pm 9) & 375( \pm 7)^{\mathrm{A}} & 796( \pm 5) & 1,123( \pm 37)^{\mathrm{A}}\end{array}$

ECs were treated with vehicle (DMSO), rapamycin $(10 \mathrm{ng} / \mathrm{ml}), \mathrm{IFN}-\gamma(50 \mathrm{ng} / \mathrm{ml})$, or rapamycin $(10 \mathrm{ng} / \mathrm{ml})$ plus IFN- $\gamma(50 \mathrm{ng} / \mathrm{ml})$ for 72 hours. Cells were harvested, labeled, and analyzed by FACS, as described in Methods. Results are expressed as corrected MFI, in arbitrary units of fluorescence, obtained by subtracting MFI of isotype control from MFI of specifically stained samples. Mean \pm SEM of triplicate samples is shown. Statistical significance was determined between vehicle and rapa and between IFN- $\gamma$ and IFN- $\gamma$ plus rapamycin. ${ }^{A} P<0.006$. the absence of effector cytokines such as IFN- $\gamma$ and IL-17, as well as the absence of inhibitory cytokines TGF- $\beta$ and IL-10 (Figure 5C). It is important to note, however, that such Tregs were also found in cocultures with control ECs; they simply represent a significantly smaller percentage of the total activated $\left(\mathrm{CD} 25^{+}\right)$ $\mathrm{T}$ cells compared with their frequency after coculture with rapa-ECs.

These experiments establish that the population of $\mathrm{CD} 25^{+} \mathrm{T}$ cells activated by rapa-ECs contains a greater percentage of Tregs. This shift in the balance could arise from an absolute expansion of Tregs, a reduction in the expansion of $\mathrm{T}$ effector cells, or both. FACS analysis suggest that the change in ratio largely resulted from a decrease in the number of effector T cells

T cells used to initiate the cocultures were depleted of preexisting $\mathrm{CD} 25^{+}$cells (reduced from about $2 \%$ of the memory $\mathrm{CD} 4^{+}$population to less than $0.02 \%$; data not shown), a pool that contains the natural Tregs, prior to coculture. In other words, the CD25+ $\mathrm{FoxP}^{+}$cells induced by coculture with rapa-ECs arise from previously CD25- cells and thus could qualify as being iTregs. However, about $4 \%-6 \%$ of human $\mathrm{CD} 25^{-}$memory $\mathrm{CD} 4^{+} \mathrm{T}$ cells in peripheral blood expressed FoxP3 (Figure 4B), and we cannot determine whether $\mathrm{CD} 25^{\text {hiFoxP }} 3^{+} \mathrm{T}$ cells arise from this population, a previously FoxP3- population, or both. Last, allogeneic memory T cells activated by mTOR-knockdown ECs similarly contained a greater percentage of FoxP3 ${ }^{+}$cells (Figure 4C), suggesting that our observations with rapa-ECs were not due to carryover of rapamycin acting directly on T cells.

In humans, FoxP3 is not an exclusive marker of Tregs, as it is also transiently expressed on activated conventional $\mathrm{CD}^{+}$ $\mathrm{T}$ cells $(48,49)$. To determine whether $\mathrm{CD} 4^{+} \mathrm{CD} 25^{\text {hi }} \mathrm{CD} 127^{\mathrm{lo}}$ FoxP $^{+}$cells induced by rapa-ECs were Tregs, we tested their functional characteristics using in vitro suppression assays. Because the frequency of alloreactive $T$ cells capable of responding to an EC donor is low (1\%), we were limited in the ability to sort sufficient numbers of cells for use in suppression assays. To bypass this obstacle, we used a system previously developed in our laboratory (50) wherein ECs are retrovirally transduced to express the extracellular domain of CD32, an Fc $\gamma \mathrm{R}$ that binds and displays IgG antibodies, and then preloaded with the antiCD3 antibody OKT3. These OKT3-loaded ECs are capable of polyclonally activating $100 \%$ of T cells. Using this system, we confirmed that $\mathrm{T}$ cells activated by anti-CD3-loaded CD32+ rapa-ECs contained higher percentages of $C D 4^{+} C D 25^{\text {hi }} C D$ $127^{\text {lo }}$ FoxP $3^{+}$cells (Figure $5 \mathrm{~A}$ ). These cells were then sorted on the basis of high CD25 expression and added to cocultures of freshly isolated CFSE-labeled T cells and OKT3-loaded ECs. Addition of these cells potently suppressed proliferation of the CFSE-labeled responder $\mathrm{T}$ cells (Figure 5A). Furthermore, analysis of bisulfite-converted DNA revealed that $\mathrm{CD}^{+}$ $\mathrm{CD} 25^{\text {hi }} \mathrm{CD} 127^{\text {lo }} \mathrm{T}$ cells activated by rapa-ECs displayed strong demethylation of the TSDR within the FOXP3 gene (Figure $5 \mathrm{~B})$. Together these data suggest that $\mathrm{FoxP}^{+}$cells activated by rapa-ECs are indeed Tregs. Analysis of the cytokines produced by these cells by intracellular cytokine staining revealed (i.e., FoxP3-) present within the $\mathrm{CD}^{-} 5^{+}$population (Figure 5D). To determine whether this change in the ratio of Tregs to $T$ effecrepeated our suppression assays using the total $\mathrm{CD} 25^{+}$population rather than focusing only on the CD25 hi subset. The net effect of this altered balance of effector versus Tregs is that the total CD25 population activated by rapa-ECs was more suppressive than the equivalent population activated by control ECs (Figure 5E).

Rapamycin alters EC expression of costimulatory molecules and secretion of IL-6. To identify specific mechanisms by which rapamycin alters EC alloimmunogenicity, we investigated how the drug affects endothelial expression of immunologically relevant molecules under basal and IFN- $\gamma$-stimulated conditions (Table 1). As expected, MHC class I was expressed under basal conditions and upregulated by IFN- $\gamma$, while MHC class II was only detected after IFN- $\gamma$ treatment. Rapamycin did not alter expression of either molecule, consistent with our observation that equal frequencies of $\mathrm{T}$ cells were initially activated by recognition of allogeneic MHC class II molecules on control ECs and rapa-ECs (Figure 1A). CD40 was downregulated on rapa-ECs, while expression of the costimulatory molecules OX40 ligand and GITR ligand was increased under both basal and IFN- $\gamma$-stimulated conditions (OX40 ligand only). Furthermore, compared with control ECs, rapa-ECs expressed significantly higher levels of the inhibitory molecules PD-L1 and PD-L2 under basal conditions and also following optimal IFN- $\gamma$ treatment. In addition to cell surface molecules, we also investigated soluble mediators released by ECs that may influence the response of allogeneic T cells. Interestingly, we found that rapamycin profoundly inhibited EC secretion of the T cell-activating cytokine IL- 6 , in response to IFN- $\gamma$ or to TNF (Figure 6A). In contrast, rapamycin only mildly reduced secretion of IL-8 in response to TNF (Figure 6A), suggesting that the reduction in IL- 6 was not due to decreased viability or a generalized block of protein secretion in rapa-ECs.

To determine which component of mTOR signaling mediated these changes, we knocked down mTOR, raptor, and rictor in ECs. Similar to rapamycin treatment, mTOR knockdown increased expression of OX40 ligand, GITR ligand, PD-L1, and PD-L2, an effect that was phenocopied by raptor, but not rictor, knockdown (Table 2). mTOR knockdown also decreased EC secretion of IL-6 in response to TNF, and interestingly, both raptor and rictor tor cells among activated $T$ cells was functionally significant, we 


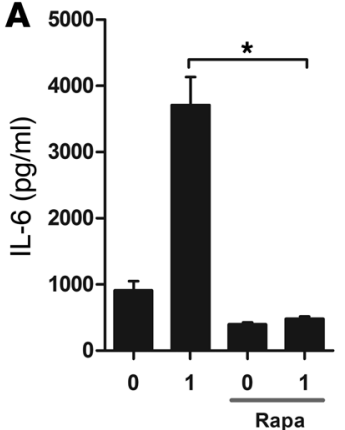

TNF (ng/ml)

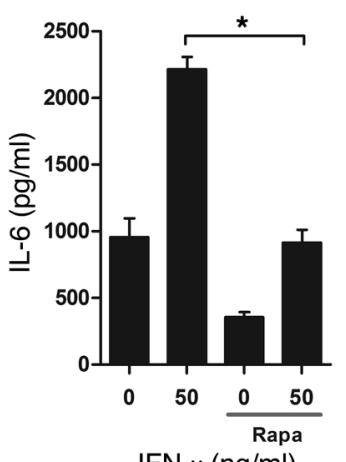

IFN- $\gamma(\mathrm{ng} / \mathrm{ml})$

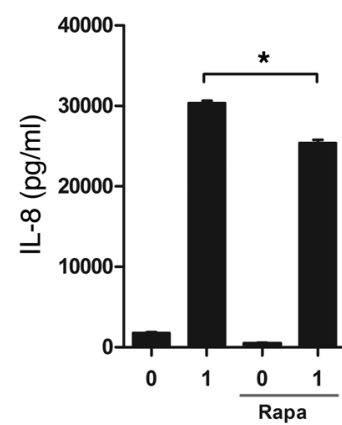

TNF (ng/ml)

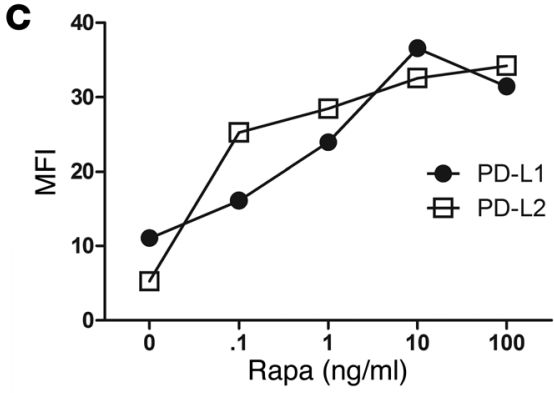

D
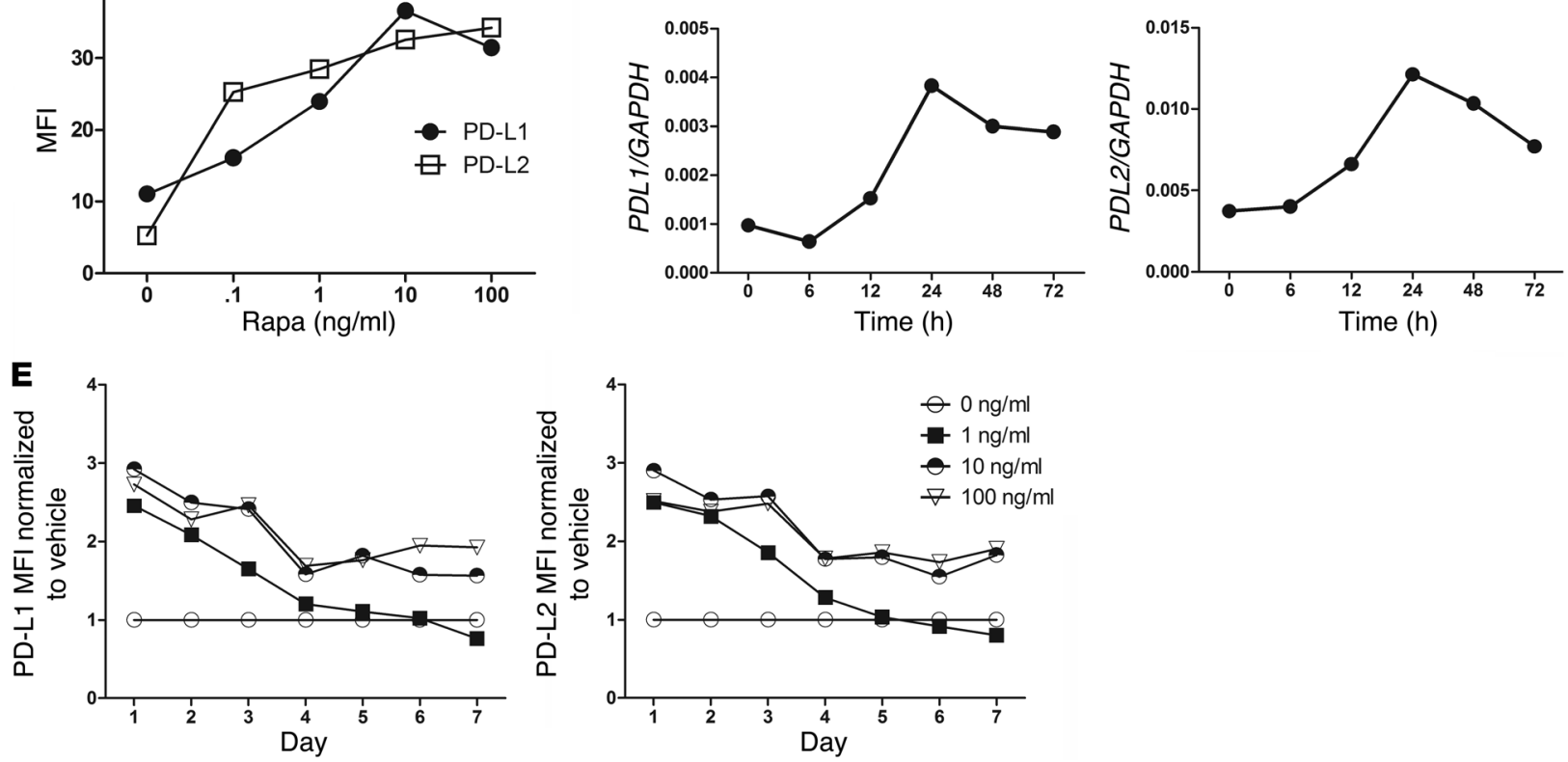

\section{Figure 6}

Rapamycin decreases EC secretion of IL-6 and increases expression of PD-L1 and PD-L2. (A) ECs were treated with vehicle or rapamycin for 2 hours, at which time the indicated doses of TNF- $\alpha$ or IFN- $\gamma$ were added to cells. After 24 hours, supernatants were assessed for IL- 6 or IL- 8 by ELISA. (B) GFP-, mTOR-, rictor-, and raptor-knockdown ECs were treated with $1 \mathrm{ng} / \mathrm{ml} \mathrm{TNF}$. After 24 hours, supernatants were assessed for IL-6. (C) ECs treated with the indicated dose of rapamycin for 72 hours were analyzed by FACS. Similar results were seen in 3 independent experiments. (D) mRNA expression in ECs treated with rapamycin for the indicated times. Transcript levels were normalized to GAPDH. Similar results were seen in 3 independent experiments. (E) ECs were treated with the indicated dose of rapamycin for 72 hours, washed, and maintained for up to 7 days (in the absence of drug) and then analyzed by FACS. MFI values are normalized to control-treated $(0 \mathrm{ng} / \mathrm{ml} \mathrm{rapa)} \mathrm{ECs.} \mathrm{Similar} \mathrm{results}$ were seen in 2 independent experiments. Mean \pm SEM of triplicate samples is shown from $2(\mathbf{A}$ and $\mathbf{B})$ independent experiments. ${ }^{*} P<0.05$.

knockdown reproduced this effect (Figure 6B). These data suggest that disruption of both mTOR signaling complexes is involved in mediating the effects of rapamycin.

$P D-L 1$ and $P D-L 2$ are induced by rapamycin and contribute to the decreased alloimmunogenicity of rapa-ECs. Of the cell surface changes induced by rapamycin (Table 1), we chose to focus on changes in PD-L1 and PD-L2, as these molecules are known to send inhibitory signals to $\mathrm{T}$ cells. Using flow cytometric analysis, we found that rapamycin increased EC cell surface expression of PD-L1 and PD-L2 in a dose-dependent manner with maximal induction occurring at 72 hours with $10 \mathrm{ng} / \mathrm{ml}$ drug (Figure 6C). Using qRT-PCR, we found that PDL1 and PDL2 mRNA transcripts were upregulated by rapamycin treatment, peaking at 24 hours but still remaining elevated at 72 hours (Figure 6D). To study the longevity of this effect, we cultured rapamycin-treated ECs in the absence of drug. With lower treatment doses, PD-L1 and PD-L2 remained elevated 4 days after withdrawal of drug, while at higher doses these molecules were still elevated at 7 days (Figure 6E). Furthermore, shRNA knockdown of mTOR increased EC surface expression of PD-L1 and PD-L2 (Table 2), implying that the effect of rapamycin was due to specific inhibition of mTOR signaling. Last, addition of PD-L1- and PD-L2-blocking antibodies into cocultures restored the ability of allogeneic memory $\mathrm{CD}^{+} \mathrm{T}$ cells to proliferate in response to rapa-ECs (Figure 7A). Because rapa-ECs secreted reduced amounts of IL-6 (Figure 6A), we investigated whether this deficit may also contribute to their weak allostimulatory ability. However, in contrast to PD-L1/PD-L2 blockade, addition of recombinant IL-6 to replace the missing cytokine did not com- 


\section{Table 2}

Flow cytometric analysis of EC cell surface costimulatory molecules on control (GFP)-, mTOR-, rictor-, and raptor-knockdown ECs

\begin{tabular}{lcccc}
\hline & GFP $($ MFI) & mTOR (MFI) & Rictor (MFI) & Raptor (MFI) \\
HLA-ABC & $1,958( \pm 10)$ & $2,550( \pm 254)$ & $2,206( \pm 100)$ & $2,215( \pm 81)$ \\
LFA-3 & $2,860( \pm 6)$ & $4,157( \pm 11)^{\mathrm{A}}$ & $3,360( \pm 21)^{\mathrm{A}}$ & $3,372( \pm 22)^{\mathrm{A}}$ \\
CD40 & $1,352( \pm 11)$ & $1,541( \pm 30)$ & $1,316( \pm 9)$ & $1,325( \pm 10)$ \\
OX40 ligand & $395( \pm 2)$ & $619( \pm 6)^{\mathrm{A}}$ & $438( \pm 16)$ & $657( \pm 3)^{\mathrm{A}}$ \\
GITR ligand & $133( \pm 3)$ & $263( \pm 2)^{\mathrm{A}}$ & $134( \pm 5)$ & $260( \pm 3)^{\mathrm{A}}$ \\
PD-L1 & $427( \pm 22)$ & $989( \pm 5)^{\mathrm{A}}$ & $413( \pm 5)$ & $853( \pm 12)^{\mathrm{A}}$ \\
PD-L2 & $997( \pm 8)$ & $1,942( \pm 11)^{\mathrm{A}}$ & $1,073( \pm 22)$ & $1,741( \pm 35)^{\mathrm{A}}$
\end{tabular}

ECs transduced with shRNA targeting GFP (control), mTOR, rictor, and raptor were harvested, labeled, and analyzed by FACS, as described in Methods. Results are expressed as corrected MFI, in arbitrary units of fluorescence, obtained by subtracting MFI of isotype control from MFI of specifically stained samples. Mean \pm SEM of triplicate samples is shown. Statistical significance was determined between vehicle and mTOR, rictor, and raptor. ${ }^{A} P<0.006$.

we can perform transplant into the second host at a time of our choosing, we can ensure that recipients will have optimal levels of circulating human T cells at transplant. This is a feature we cannot otherwise control, since circulating $\mathrm{T}$ cell numbers require 1-2 weeks to reach adequate levels but begin to cause pathological changes in the host after 4-5 weeks, and we do not know in advance when human artery segments will become available. This design also provides a closer recapitulation of clinical transplantation, where allografts are transplanted into donors with circulating T cells in a "physiological" range.

To test whether rapamycin pretreatment has a protective role in vivo, we treated mice with healed artery grafts with vehicle or rapamycin for 72 hours, at which

pensate for the rapamycin effect, as allogeneic $T$ cells still poorly proliferated in response to rapa-ECs (Figure 7B). Collectively, these data suggest that rapamycin reduces EC alloimmunogenicity, at least in part, by increasing cell surface expression of PD-L1 and PD-L2. The reduction in IL-6 does not appear to have been functionally significant in these in vitro assays.

It has been previously shown that PD-L1 and PD-L2 on APCs mediate peripheral tolerance and can enhance generation of Tregs (51). Additionally, the presence of IL-6 is known to antagonize the function and formation of Tregs (52). Since rapa-ECs have elevated expression of PD-L1 and PD-L2 and poorly secrete IL-6, we explored whether either of these effects contributed to the increased percentages of Tregs activated by rapa-ECs. We found that addition of recombinant IL- 6 into cocultures had little effect on the percentage of $\mathrm{FoxP}^{+}$cells (Figure 7C). In contrast, serological blockade of PD-L1 and PD-L2 partially reversed the increased percentage of FoxP $3^{+}$cells among activated $\mathrm{T}$ cells in rapa-EC versus control EC cocultures (Figure 7D). Thus, we conclude that the elevated expression of PD-L1 and PD-L2 on rapa-ECs significantly contributed to the preferential activation of CD $25^{+} \mathrm{FoxP} 3^{+}$cells.

Rapamycin pretreatment reduces $T$ cell-mediated injury in buman artery allografts. To investigate whether pretreatment of allograft ECs with rapamycin can provide protective effects in vivo, we used a human-mouse chimeric model of artery allograft rejection recently developed by our laboratory (53). In this model, short segments of human artery are implanted into the infrarenal aortae of immunodeficient SCID/beige mice and left to heal for 30 days, at which point the graft is harvested and re-transplanted into a second SCID/beige mouse previously receiving adoptive transferred of human PBMCs allogeneic to the artery graft. In these mice, adoptive transfer of human PBMCs yields stable engraftment of human $\mathrm{CD}^{+} \mathrm{T}$ cells capable of mediating graft rejection but does not result in engraftment of human myeloid (monocytes, macrophages, DCs, neutrophils) or NK cells. This re-transplant design was selected for three reasons. First, it assures that only the artery is exposed to drug, avoiding confounding effects of rapamycin on $\mathrm{T}$ cells. Second, by allowing the arteries to quiesce in the primary mouse host, the model eliminates variability (injury status, time from harvest/spent on ice) in the state of human arteries at the time the arteries first encounter allogeneic $T$ cells, which could influence the subsequent $\mathrm{T}$ cell alloresponse. And third, because time grafts were harvested, perfused, and re-transplanted into PBMC-inoculated mice. Because these secondary recipients are not exposed to rapamycin, the system allows us to determine the effects of rapamycin on graft alloimmunogenicity, independent of any direct effects on the circulating human lymphocytes. Furthermore, at the time of re-transplant, the human artery graft consists of human luminal ECs, medial (and rare intimal) human smooth muscle cells, human adventitial fibroblasts and microvessels, but no detectable human (passenger) leukocytes (54). In other words, human ECs are the only functional allogeneic APCs in the graft. Using this model, we found that rapamycin pretreatment of grafts reduced the total number of $\mathrm{T}$ cells infiltrating into the vessel intima (Figure $8 \mathrm{~A}$ ) and concomitantly reduced both the total vessel area and intima expansion (Figure 8B) at 10 days following re-transplant. At this early stage, there was no loss of lumen in either group, as the outward expansion of the control vessels compensated for intimal expansion (Figure 8B and ref. 55). To determine the durability of this protective effect, we extended our experiments to 3 weeks following re-transplantation. At this later time, we found that rapamycin-pretreated grafts still had reduced total vessel areas and intima sizes compared with control grafts (Figure 8C); however, the magnitude of this effect appeared to be diminished relative to earlier time points. Together, these data suggest that pretreatment of allografts with rapamycin can reduce subsequent $\mathrm{T}$ cell-mediated rejection, although the effect may wane with time.

Based on our observations that rapamycin reduces the alloimmunogenicity of cultured ECs via induction of PD-1 ligands, we examined graft expression of these molecules via immunofluorescence. We found that rapamycin-pretreated grafts, harvested 10 days after re-transplant into PBMC-inoculated mice, had pronounced EC expression of both PD-L1 and PD-L2 (Figure 9A). These molecules were barely detectable on the ECs lining replicate control-treated grafts re-transplanted into paired mice that had been adoptively transferred with the same human PBMCs. To determine whether this induced expression of PD-L1 and PD-L2 could be contributing to the protective effects observed in rapamycin-pretreated grafts, we transplanted paired rapamycin-pretreated grafts into pairs of PBMC-inoculated mice and treated these animals with either a neutralizing $\mathrm{F}\left(\mathrm{ab} \mathrm{b}^{\prime}\right)_{2}$ fragment to $\mathrm{PD}-1$ or an isotype-matched $\mathrm{F}\left(\mathrm{ab} \mathrm{b}^{\prime}\right)_{2}$ for 14 days. (This anti-PD-1 F[ab'] $]_{2}$ fragment effectively reduced the 

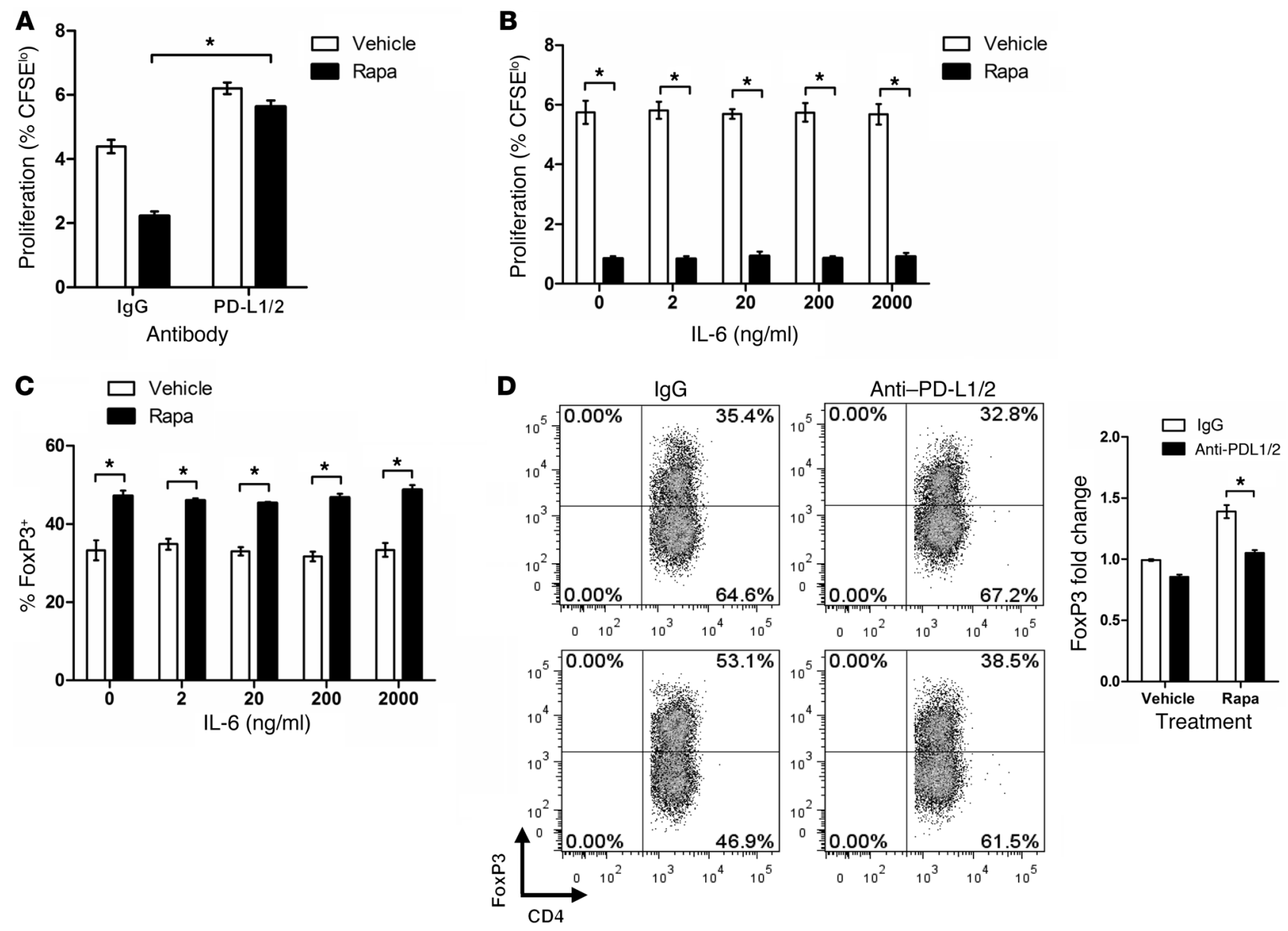

Figure 7

Elevated expression of PD-L1 and PD-L2, but not hyposecretion of IL-6, contributes to altered alloimmunogenicity of rapa-ECs. (A) Control ECs and rapa-ECs were cocultured with CFSE-labeled allogeneic CD4+ memory T cells in the presence of blocking antibodies to PD-L1 and PD-L2, or isotype control. Proliferation of T cells was determined after 7 days via CFSE dilution. (B) Control ECs and rapa-ECs were cocultured with CFSE-labeled allogeneic CD4+ memory T cells in the presence of indicated dose of recombinant IL-6. Proliferation of T cells was determined after 7 days via CFSE dilution. (C) Intracellular FACS analysis of allogeneic CD4+ memory T cells cocultured with control or rapa-ECs in the presence of the indicated dose of recombinant IL-6. Numbers represent percent CD4+FoxP3 ${ }^{+}$cells in the CD4 ${ }^{+}$CD25 $5^{+}$gate. (D) Intracellular FACS analysis of allogeneic CD4+ memory T cells cocultured with control or rapa-ECs in the presence of blocking antibodies to PD-L1 and PD-L2, or isotype control. Representative FACS plots are gated on CD4+CD25+ T cells. Data are normalized to FoxP3 expression in the control group (vehicle with isotype $\lg$ G). Mean \pm SEM of triplicate samples is shown from $3(\mathbf{A}$ and $\mathbf{D})$ or 2 (B and $\mathbf{C})$ independent experiments. ${ }^{\star} P<0.05$.

protective effect of rapamycin pretreatment of cultured ECs [data not shown].) We found that PD-1 blockade increased the vessel area and intima size of rapamycin-pretreated grafts (Figure 9B), as well as the number of infiltrating intimal $\mathrm{T}$ cells (Figure 9C), compared with animals treated with the isotype control. We conclude that PD-1 ligands contribute to the protection of rapamycin-pretreated grafts against $\mathrm{T}$ cell-mediated injury in vivo.

\section{Discussion}

We found that the mTOR inhibitor rapamycin modulates the alloimmunogenicity of human ECs. Specifically, rapa-ECs poorly stimulate alloreactive memory $\mathrm{CD}^{+} \mathrm{T}$ cells to proliferate and secrete effector cytokines without affecting recognition of MHC class II molecules on the ECs (assessed by induction of CD25). Similar effects were observed with ECs in which mTOR had been knocked down by shRNA. The recognition of MHC class II is significant, because $\mathrm{T}$ cells that had been cultured with rapaECs became hyporesponsive to restimulation by ECs from the same donor, an effect largely abrogated by blocking MHC class II recognition in the primary culture. Additionally, $\mathrm{T}$ cells activated by rapa-ECs contained an increased ratio of suppressive $\mathrm{CD} 4^{+}$ CD25 ${ }^{\text {hi }} \mathrm{CD} 127^{\text {lo }} \mathrm{FoxP}^{+}$Tregs to $\mathrm{CD} 4^{+} \mathrm{CD} 25^{+} \mathrm{FoxP}^{-}$effector $\mathrm{T}$ cells. Mechanistically, rapamycin treatment (or mTOR knockdown) altered endothelial expression of several costimulatory molecules, but not MHC molecules, and reduced EC secretion of the proinflammatory cytokine IL- 6 in response to IFN- $\gamma$ and TNF. The primary effect on the $\mathrm{CD}^{+} \mathrm{T}$ cell responses appears to be mediated via induction of PD-L1 and PD-L2 on rapa-ECs, an effect that appears to be much more substantial than the previously described upregulation of these molecules in response to IFN- $\gamma$. In vivo, in a human-mouse chimeric model, pretreatment of human artery allografts with rapamycin led to upregulation of 
A
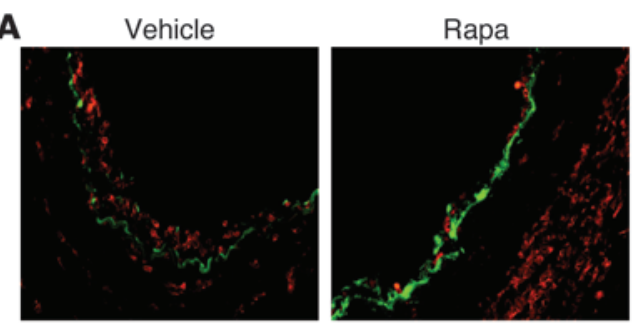

B

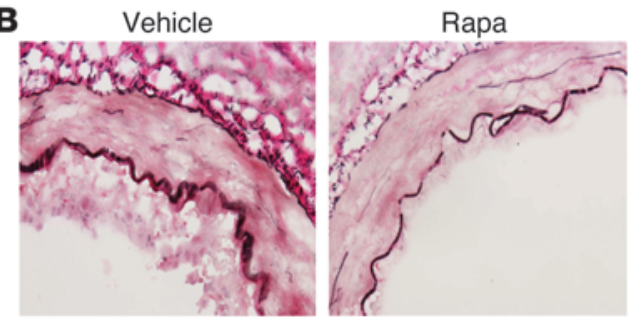

C

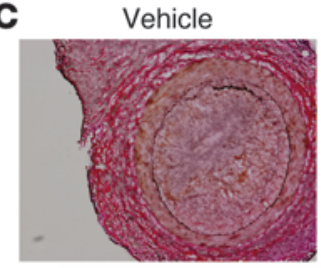

Rapa

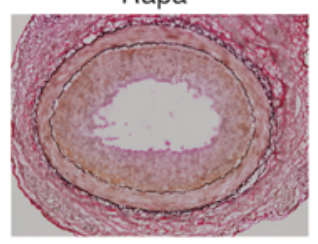

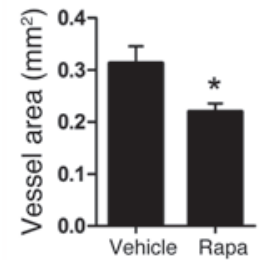

Treatment

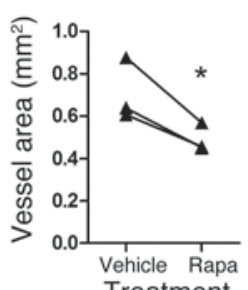

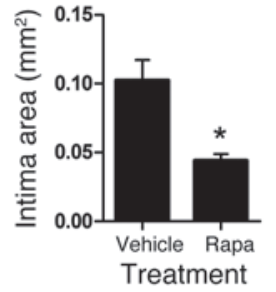
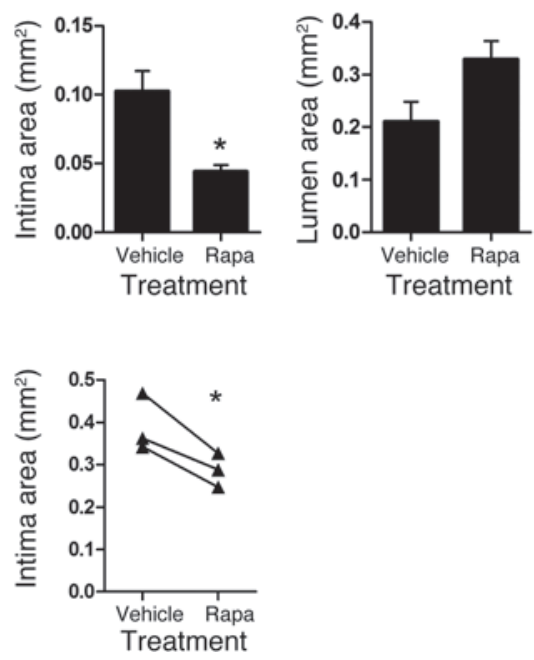

Figure 8

Pretreatment of human arterial allografts with rapamycin reduces T cell-mediated graft injury in vivo. (A) Human artery interposition grafts were pretreated with vehicle or rapamycin and then re-transplanted into PBMC-reconstituted hosts and harvested after 10 days. Representative immunofluorescence detection of infiltrating $C D 3^{+} T$ cells in graft vessel intima. ECs are stained with Ulex and visualized in green; $C D 3^{+}$cells are visualized in red. Intimal T cells were counted. Original magnification, $\times 200$. (B) Arterial graft sections (treated as above) were stained with EVG and the total vessel area (area bounded by external elastic lamina and lumen), intimal area (area bounded by internal elastic lamina and lumen), and lumen area quantified using ImageJ. Original magnification, $\times 200$. (C) As in B, except grafts were harvested 3 weeks after re-transplant into PBMC-reconstituted hosts. Original magnification, $\times 100$. Mean \pm SEM is shown for $\mathbf{A}$ and $\mathbf{B}\left(n=4\right.$ per group) and $\mathbf{C}\left(n=3\right.$ per group). ${ }^{*} P<0.05$.

PD-L1 and PD-L2 on graft ECs and protected allografts, for up to 3 weeks, against subsequent $\mathrm{T}$ cell-mediated infiltration and rejection, while blockade of PD-1 worsened immune injury in rapamycin-pretreated grafts.

The exclusive use of human materials in our studies provides two major advantages over murine systems. First, human ECs can activate resting allogeneic effector memory $\mathrm{CD}^{+} \mathrm{T}$ cells (10), whereas mouse ECs only activate CD4 ${ }^{+}$Tregs (56). Given their expression of MHC molecules and their direct contact with the host circulation, vascularized graft ECs are the first donor-derived cells to encounter and interact with the recipient immune system. Thus, it is likely that the EC-CD4+ $4^{+}$cell interaction is important in human allogeneic responses, and strong evidence suggests that such an interaction plays a pathologic role in the development of cardiac allograft vasculopathy (18, $57,58)$. Second, human transplant recipients have high numbers of alloreactive memory $\mathrm{T}$ cells, which are not present in the circulation of rodents housed under pathogen-free conditions (14). Alloreactive memory T cells act as important mediators of rejection and are believed to be more resistant to immune suppression and tolerance induction (15). Because our model reproduces both of these phenomena, which are specific to human transplantation and commonly overlooked in rodent studies, we propose that our data can more accurately predict clinical outcomes than can results from conventional mouse models.

The mTOR inhibitor rapamycin was initially believed to act as an immunosuppressant by directly blocking proliferation of $\mathrm{T}$ lymphocytes. Given that mTOR is ubiquitously expressed, recent data demonstrating that rapamycin has effects on other cell types is not surprising $(43,59)$. Both human and murine DCs differentiated in the presence of rapamycin poorly stimulate allogeneic $\mathrm{T}$ cells and express low levels of MHC class I, MHC class II, CD80, and CD86 $(45,46)$. We provide the first evidence to our knowledge that rapamycin reduces the alloimmunogenicity of human ECs. In contrast to DCs, human ECs do not express CD80 or CD86, and rapamycin did not alter EC expression of MHC molecules, but did lead to increased expression of PD-L1 and PD-L2, associated with reduced allostimulatory capacity. PD-L1 and PD-L2 are inhibitory molecules that bind the PD-1 receptor, expressed on activated T cells (60). Upon ligation by either PD-L1 or PD-L2, PD-1 antagonizes TCR signaling via recruitment of SHP-1 and SHP-2 phosphatases that inhibit phosphorylation of CD3ל, ZAP70, and PKCO $(51,61)$. PD-1 ligation also inhibits PI3K activity and downstream activation of Akt (36) and mTOR. Together, these signaling events reduce proliferation 
$\mathbf{A}$

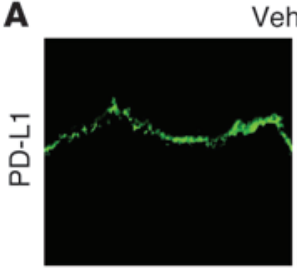

Vehicle
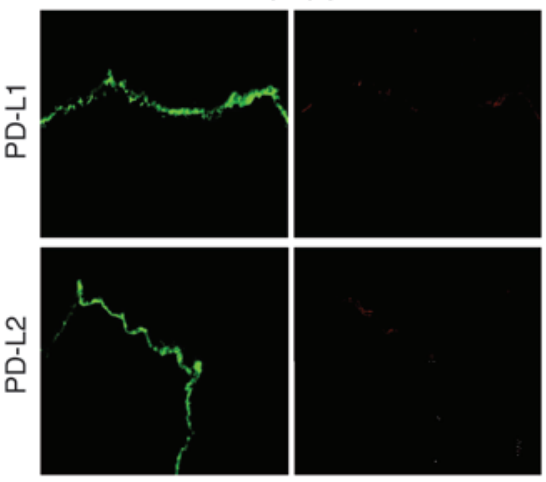
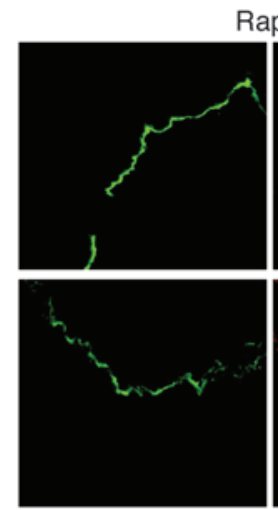

Rapa

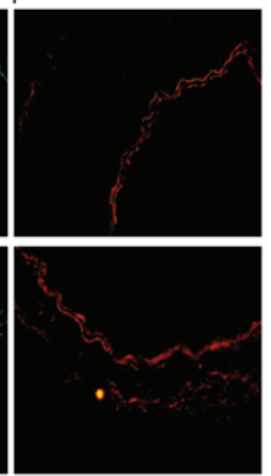

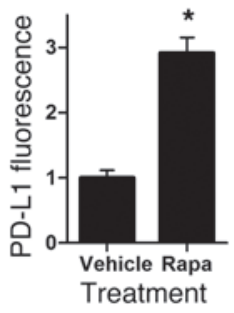

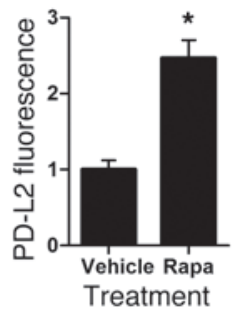

B

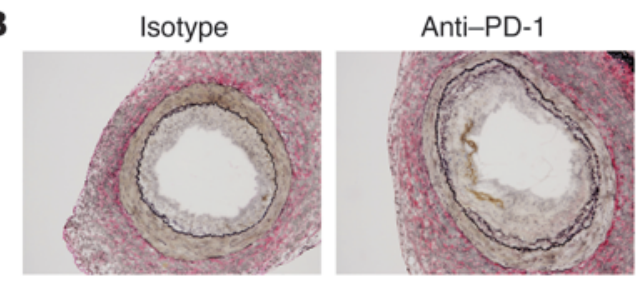

c

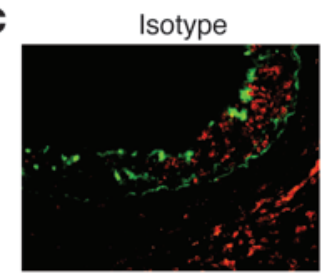

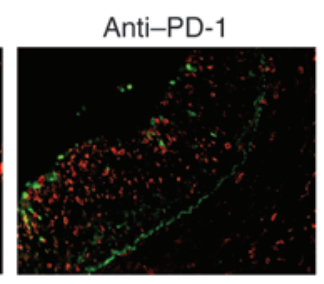
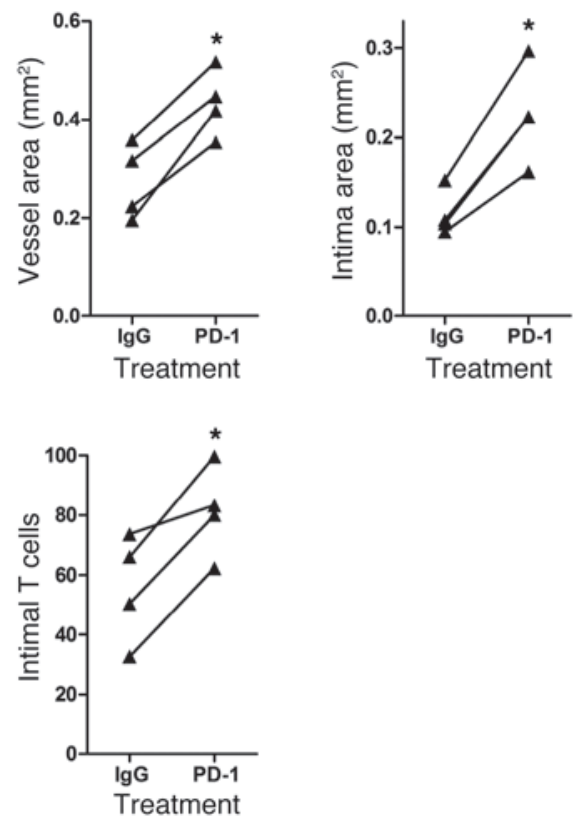

Figure 9

PD-1 ligands protect rapamycin-pretreated grafts from T cell-mediated graft injury in vivo. (A) Human artery interposition grafts were pretreated with vehicle or rapamycin and then re-transplanted into PBMC-reconstituted hosts and harvested after 10 days. Representative immunofluorescence detection of PD-L1 and PD-L2 on graft ECs is shown. ECs are stained with Ulex and visualized in green; PD-L1 and PD-L2 are visualized in red. Quantification of PD-L1 and PD-L2 immunofluorescence is normalized to staining in vehicle-pretreated grafts. Original magnification, $\times 200$. (B) Rapamycin-pretreated grafts were transplanted into PBMC-reconstituted mice and subsequently treated with neutralizing PD-1 F $\left(a b^{\prime}\right)_{2}$ or control $F\left(a b^{\prime}\right)_{2}$ fragments. Grafts were harvested after 2 weeks and stained with EVG. Original magnification, $\times 100$. (C) Representative immunofluorescence detection of infiltrating CD3+ T cells in grafts treated as in B. ECs are stained with Ulex and visualized in green; $\mathrm{CD}^{+}$cells are visualized in red. Intimal T cells were counted. Original magnification, $\times 200$. Mean \pm SEM are shown for A-C $\left(n=4\right.$ per group). ${ }^{*} P<0.05$.

and cytokine secretion in activated T cells. In allogeneic heart (62), corneal (63), and skin (64) transplant models, blockade or absence of PD-1 signaling accelerated rejection, while administration of PD-L1.Ig (a PD-1 agonist) reduced cardiac allograft vasculopathy (65). Collectively, these data suggest a role for PD-1 ligands in maintaining allograft tolerance. Thus, the induction of PD-1 ligands on allograft ECs, by rapamycin, would be expected to provide protective effects by downregulating host alloreactive effector $\mathrm{T}$ cells.

Rapamycin inhibits EC secretion of IL- 6 in response to IFN- $\gamma$ and TNF. IL- 6 is a proinflammatory cytokine that has numerous effects on $T$ cell activation, differentiation, and recruitment (66-68). In the context of transplantation, increased levels of
IL-6 correlated with adverse outcomes in human allograft recipients $(69,70)$. IL-6 is known to inhibit the function of natural Tregs (71), as well as the induction of iTregs (52). Given that neutralizing IL-6 in such cocultures increases Treg formation (5) and that rapa-ECs poorly secrete IL-6, we were surprised to find that addition of recombinant IL-6 into cocultures did not alter the proportions of FoxP3 $3^{+}$cells. We suspect that the explanation resides in the observation that rapamycin treatment did not completely eliminate IL- 6 production by the ECs in our culture system. Nevertheless, the downregulation of IL- 6 release could be important in the in vivo setting, where, due to perfusion, local cytokine levels may be more limited. 
In rodent models, infusion of either natural or inducible Tregs into recipients prior to transplantation prevented rejection of allografts (72). In humans, increased numbers of Tregs are found in the circulation and within allografts of recipients that have achieved immunosuppression-free graft survival (operational tolerance) $(1,73,74)$. We found that allogeneic $\mathrm{T}$ cells activated by rapa-ECs contained a higher percentage of suppressive $\mathrm{CD}^{+}$ $\mathrm{CD} 25^{\text {hi }} \mathrm{CD} 127^{\text {lo }} \mathrm{FoxP} 3^{+} \mathrm{T}$ cells, and such cells displayed significant demethylation of the TSDR within the FOXP3 locus. While the increase in $\mathrm{FoxP}^{+}$cells activated by rapa-ECs compared with control ECs (Figure 4B) may appear modest, it is not known how many of these FoxP3 ${ }^{+}$cells actually represent suppressive Tregs, since conventional human T cells transiently express FoxP3 upon activation. However, we demonstrate that this increase is functionally significant, as the $\mathrm{CD} 25^{+} \mathrm{T}$ cell pool primed by rapa-EC was far more suppressive than control, especially at lower Treg/responder ratios (Figure $5 \mathrm{E}$ ). Since our highly purified $\mathrm{CD} 4^{+}$memory $\mathrm{T}$ cells are depleted of preexisting $\mathrm{CD} 25^{+} \mathrm{T}$ cells, a subpopulation that in humans contains natural Tregs, one may consider the FoxP3 ${ }^{+}$ cells induced by rapa-ECs as iTregs. The fundamental effect of rapamycin treatment appears to be a loss of the capacity of rapaECs to activate effector cells, resulting in an increase in the ratio of regulatory to effector $T$ cell populations. We demonstrate that PD-1 ligands partially contribute to this effect, as their blockade reduces the preferential activation of $\mathrm{CD} 25^{+} \mathrm{FoxP} 3^{+}$cells by rapaECs. Importantly, we show that shRNA knockdown of mTOR in ECs phenocopies the increased ability of rapa-ECs to favor Treg activation, thereby ruling out the possibility that carryover of rapamycin may be directly inducing Tregs. The enhanced ability of rapamycin-treated allograft ECs to activate Tregs would be beneficial in the context of transplantation by reducing rejection and promoting tolerance.

Last, using a human-mouse chimeric model of allograft artery rejection, we observed that pretreatment of arterial allografts with rapamycin diminished $\mathrm{T}$ cell-mediated graft injury, as assessed by $\mathrm{T}$ cell intimal infiltration and intimal expansion. These protective effects coincided with increased expression of PD-L1 and PD-L2 on treated graft ECs. The induced expression of PD-1 ligands was detectable 10 days after re-transplant, suggesting durability of this response. We cannot rule out that the $\mathrm{T}$ cells present in the host animal, perhaps through production of IFN- $\gamma$, contributed to this prolonged expression; however, rapamycin-inducible expression was durable in cultured ECs and greater than that induced by IFN- $\gamma$, and little if any expression was observed in grafts not pretreated with rapamycin, despite a greater number of infiltrating $T$ cells. To elucidate the role of these molecules, we serologically blocked PD-1 in animals transplanted with rapamycin-pretreated grafts and found that the resulting vessels had increased areas and intima sizes, as well as more infiltrating intimal $\mathrm{T}$ cells when compared with animals treated with an isotype control. These data suggest that rapamycin-induced PD-1 ligands contribute to the protection of rapamycin-pretreated grafts against immune-mediated rejection. Based on our data with cultured ECs, we speculate that this induced expression of PD-1 ligands on graft ECs acts to reduce their alloimmunogenicity and may lead to preferential activation of Tregs in vivo. In addition, we have recently shown that serological neutralization of IL- 6 diminishes intimal expansion in a similar model of artery allograft rejection (5). Given that rapamycin reduces IL-6 production by cultured ECs, it is possible that such a phenome- non may further contribute to the protective effects of rapamycin in vivo. Overall we conclude that pretreatment of allografts with rapamycin downmodulates human $T$ cells responses to allogeneic artery grafts in vivo. We envision that our data may be translated clinically by including rapamycin in the preservation fluid that is flushed or perfused through an explanted organ, or alternatively, by treating brain-dead cadaver donors with rapamycin prior to organ harvest. However, we caution that such an intervention requires additional preclinical study, as rapamycin has also been reported to have proinflammatory effects on monocytes, DCs, and $\mathrm{CD}^{+} \mathrm{T}$ cells $(75,76)$. If such effects on leukocytes prove to be significant, then rapamycin pretreatment could be coupled to pre-transplant depletion of donor leukocytes to eliminate donor myeloid and lymphoid cells from allografts.

The data presented in this report raise several interesting questions. Presently, we do not fully understand the specific signaling events downstream of mTOR that control EC alloimmunogenicity. In the cell, mTOR exists in two distinct signaling complexes, known as mTORC1 and mTORC2, which have differing upstream inputs and downstream outputs (77). Our data show that disruption of mTORC1 signaling (by raptor knockdown) caused changes in EC surface molecules similar to those induced by rapamycin, and such ECs poorly stimulated allogeneic T cells. In addition, we have found that inhibition of both MTORC1 and mTORC2 signaling reduces the ability of ECs to secrete IL- 6 in response to TNF. We are currently investigating whether any of the known mTORC1 and mTORC2 targets are involved in mediating these effects. Additionally, because blockade of PD-1 ligands only partially reduced the ability of rapa-ECs to preferentially activate Tregs, it is likely that there are other mechanisms behind this observation. Although ligation of GITR on Tregs by GITR ligand was initially thought to abrogate their suppressive activity (78), recent studies suggest that GITR ligand may act to maintain and expand Tregs (79-81). Additionally, the interaction between OX40 and OX40 ligand has been shown to be important in Treg cell development in the gut (82), and an OX40 agonist enhanced Treg proliferation (83). Given that both GITR ligand and OX40 ligand are highly expressed on rapa-ECs, we are exploring whether either of these molecules contributes to the ability of rapa-ECs to activate Tregs.

\section{Methods}

Isolation and culture of buman cells. PBMCs were isolated by density centrifugation of leukapheresis products obtained from anonymized healthy adult volunteers using Lymphocyte Separation Medium (MP Biomedicals). PBMCs were stored in liquid nitrogen in 10\% DMSO/90\% FBS until ready for use.

$\mathrm{CD}^{+} \mathrm{T}$ cells were isolated from PBMCs by positive selection using Dynabeads (Invitrogen) magnetic beads and released using DETACHaBEAD, according to the manufacturer's instructions. Activated T cells, preexisting Tregs, and monocytes were removed by incubating the isolated $\mathrm{CD}^{+} \mathrm{T}$ cells with $5 \mu \mathrm{g} / \mathrm{ml}$ mouse anti-CD14 (BioLegend), antiCD25 (eBioscience), and anti-HLA-DR (LB3.1; gift of J. Strominger, Harvard University, Cambridge, Massachusetts, USA) antibodies for 20 minutes, followed by depletion using magnetic pan-mouse IgG beads (Invitrogen). Memory CD4 ${ }^{+} \mathrm{T}$ cells were isolated following further negative selection using mouse anti-CD45RA (eBioscience) and pan-mouse IgG beads. Isolates were routinely $>99 \% \mathrm{CD}^{+} \mathrm{CD}^{2} 5^{-} \mathrm{HLA}-\mathrm{DR}{ }^{-} \mathrm{CD} 45 \mathrm{RA}^{-}$ by flow cytometry. It is important to note that these populations are not only devoid of activated memory $\mathrm{T}$ cells, but are also depleted of CD25-expressing natural Tregs. 
Human ECs were isolated from umbilical cords following collagenase digestion and serially cultured on $0.1 \%$ gelatin-coated tissue culture plates in M199 (Invitrogen) supplemented with 20\% FBS, L-glutamine (2 mM), penicillin $(100 \mathrm{U} / \mathrm{ml})$, streptomycin $(100 \mu \mathrm{g} / \mathrm{ml}), 0.1 \%$ EC growth supplement (Collaborative Biomedical Research), and porcine heparin $(100 \mu \mathrm{g} / \mathrm{ml}$; Sigma-Aldrich). EC cultures were used at passage levels 2-5, at which time the cultured cells are uniformly positive for the EC marker CD31 and are devoid of $\mathrm{CD} 45^{+}$contaminating leukocytes.

In vitro rapamycin treatment. ECs retrovirally transduced to express CIITA were grown on $10-\mathrm{cm}$ gelatin-coated plates and allowed to reach confluence. Post-confluent ECs were treated with varying doses (up to $100 \mathrm{ng} / \mathrm{ml}$ ) of rapamycin (Sigma-Aldrich) or vehicle (DMSO) for up to 72 hours. Fresh medium with rapamycin was added every 24 hours. Vehicle- and rapamycin-treated ECs were then washed 3 times with HBSS, collected by trypsinization, washed an additional 3 times with HBSS, and plated into 24-well plates. Viable (assessed by Trypan blue staining) control ECs and rapa-ECs were counted to ensure that equal numbers of cells were plated. In other experiments, post-confluent ECs grown in $10-\mathrm{cm}$ plates were treated with rapamycin and $50 \mathrm{ng} / \mathrm{ml}$ recombinant human IFN- $\gamma$ simultaneously for 72 hours, then plated as above.

$T$ cell activation in vitro. $\mathrm{CD}^{+}$memory $\mathrm{T}$ cell activation by allogeneic ECs requires recognition of non-self MHC class II molecules, principally HLADR, on the ECs. To reinduce EC expression of MHC class II, which is lost on serially cultured cells, ECs were retrovirally transduced with the CIITA transactivator, as previously described (50). In other experiments, expression was restored by treating ECs with $50 \mathrm{ng} / \mathrm{ml}$ recombinant human IFN- $\gamma$ (Invitrogen) for 72 hours. For in vitro stimulation, MHC class II $^{+}$ECs were washed 3 times with HBSS, plated into gelatin-coated 24-well plates, and allowed to reach confluence. $2 \times 10^{6}$ allogeneic memory $\mathrm{CD} 4^{+} \mathrm{T}$ cells were added to each well in RPMI 1640 (Invitrogen), supplemented with 10\% FBS, L-glutamine $(2 \mathrm{mM})$, penicillin $(100 \mathrm{U} / \mathrm{ml})$, and streptomycin $(100 \mu \mathrm{g} / \mathrm{ml})$. To assess proliferation, T cells were labeled with $250 \mathrm{nM} \mathrm{CFSE} \mathrm{(Molecular}$ Probes, Invitrogen) prior to coculture. Proliferation was assessed on day 7 via CFSE dilution, as previously described (84). In some experiments, recombinant human IL-6 (BioLegend) or $10 \mu \mathrm{g} / \mathrm{ml}$ of blocking antibodies to PD-L1 and PD-L2 (BioLegend) was added into allogeneic cocultures.

For restimulation experiments, unlabeled memory $\mathrm{CD}^{+}{ }^{+} \mathrm{T}$ cells were cocultured with MHC class $\mathrm{II}^{+}$allogeneic control ECs or rapa-ECs for 72 hours, collected, washed 3 times in HBSS, and rested for 3 days in the presence of $10 \mathrm{U} / \mathrm{ml}$ recombinant human IL-2 (Invitrogen), which serves as a survival factor. Recovered T cells were washed in HBSS, labeled with $250 \mathrm{nM}$ CFSE, and restimulated with either fresh MHC class $\mathrm{II}^{+} \mathrm{ECs}$ from the same donor as used in primary stimulation or from third-party donors. Proliferation was assessed (via CFSE dilution) at day 3 for T cells restimulated by matched EC donors and day 5 for T cells restimulated by thirdparty EC donors. In some experiments, blocking antibodies $(30 \mu \mathrm{g} / \mathrm{ml})$ to HLA-DR or isotype control $\operatorname{IgG}(30 \mu \mathrm{g} / \mathrm{ml})$ were added to the primary stimulation cocultures.

FACS analysis. To analyze ECs, cells were suspended with trypsin-versene, washed in PBS, and stained (on ice) in PBS supplemented with $1 \%$ BSA with antibodies against human HLA-A,B,C-FITC, HLA-DR-FITC, or CD40-PE (all from Beckman Coulter), LFA-3-PE, PD-L1-PE, or PD-L2-PE (all from BD Biosciences), OX40 ligand-PE or GITR ligand-PE (from R\&D Systems), or ICOS ligand-PE (from BioLegend).

To analyze effects on T cells, cells were collected from cocultures via vigorous pipetting, washed in PBS, and stained (on ice) in PBS supplemented with $1 \%$ BSA with antibodies against human CD4-FITC or-Pacific blue, or CD25-PE (from BD Biosciences) or CD127-PerCP-Cy5.5 (from eBioscience). For FoxP3 staining, $\mathrm{T}$ cells were first stained with anti-human CD4, CD25, CD127 fluorescent antibodies and washed in PBS. These surface-stained cells were then permeabilized and restained using a FoxP3 Staining Kit (eBioscience), according to the manufacturer's instructions, with anti-human FoxP3-APC (clone PCH101; eBioscience). For intracellular cytokine staining, $\mathrm{T}$ cells cocultured with allogeneic ECs for 6 days were stimulated for 6 hours with $40 \mathrm{ng} / \mathrm{ml} \mathrm{PMA}$ and $2 \mu \mathrm{M}$ ionomycin in the presence of Brefeldin A (eBioscience). Stimulated T cells were surface stained with anti-human CD4, CD25, CD127 fluorescent antibodies, then permeabilized using FoxP3 Staining Kit and stained with FoxP3-APC and IL-17-FITC (eBioscience), IL-10-FITC (eBioscience), IFN- $\gamma$-FITC (eBioscience), or TGF- $\beta /$ LAP-FITC (BioLegend). To ensure antibodies were functional, positive staining was analyzed in the following controls: $\mathrm{CD} 14^{+}$ monocytes stimulated with LPS (IL-10), CD4 ${ }^{+} \mathrm{T}$ cells stimulated by MHC $\mathrm{II}^{+} \mathrm{ECs}$ (IFN- $\gamma$ and IL-17), and CD4 ${ }^{+} \mathrm{T}$ cells stimulated with anti-CD 3 and anti-CD28 in the presence of IL-2 (LAP). All stained cells were analyzed on an LSR II (BD Biosciences) using FlowJo software (Tree Star).

In vitro suppression assays. The frequency of $\mathrm{T}$ cells that recognize allogeneic cells is too limited to generate significant numbers of responder cells in a primary culture. Therefore, to determine whether ECs could generate iTregs, we first modified EC stimulators to serve as polyclonal activators of $\mathrm{T}$ cell responses by retroviral transduction of the extracellular domain of Fcy receptor IIA (CD32), as previously described (50). CD32+ ECs were treated with vehicle (DMSO) or rapamycin and plated into 24-well plates, as described above. Treated ECs were then loaded with $2.5 \mu \mathrm{g} / \mathrm{ml}$ anti-CD3 (OKT3; BioLegend) for 4 hours and washed 3 times with RPMI complete medium. $2 \times 10^{6}$ allogeneic memory $\mathrm{CD}^{+} \mathrm{T}$ cells were added to each well. After 72 hours of stimulation, T cells were collected, washed, stained with CD4-FITC (Beckman Coulter) and CD25-APC (BD Biosciences), and sorted on a FACSAria (BD Biosciences) to isolate total $\mathrm{CD} 4{ }^{+} \mathrm{CD} 25^{+}$or $\mathrm{CD} 4{ }^{+} \mathrm{CD} 25^{\text {hi }}$ populations. Sorted populations were then added into cocultures of freshly isolated CFSE-labeled CD4 ${ }^{+}$memory T cells and untreated OKT3-loaded ECs at indicated suppressor/responder ratios. Proliferation of CFSE-labeled responder T cells was assessed after 4 days via CFSE dilution, a time point at which responder cells that had divided could be readily distinguished from both labeled cells that had not divided and unlabeled Tregs. Percent suppression was calculated as follows: ([proliferation of responders alone - proliferation of responders with Tregs]/proliferation of responders alone) $\times 100$ ).

qRT-PCR analysis. ECs treated with rapamycin for up to 72 hours were collected at various time points by trypsinization, lysed, and used to make cDNA using a Cells-to-CT kit (Ambion), according to the manufacturer's instructions. For $\mathrm{T}$ cell experiments, memory $\mathrm{CD} 4^{+} \mathrm{T}$ cells stimulated by allogeneic ECs for 72 hours were collected, washed, stained with fluorescent antibodies, and FACS sorted for $\mathrm{CD} 4^{+} \mathrm{CD} 25^{+}$cells. cDNA was made from these cells using the Cells-to-CT kit. All qRT-PCR reactions were assembled with TaqMan $2 \times$ PCR Master Mix and predeveloped gene expression probes from Applied Biosystems. Samples were analyzed on a CFX96 RealTime system using CFX Manager Software (Bio-Rad). Gene expression levels were normalized to GAPDH or CD3, as indicated. All Taqman probes were purchased from Applied Biosystems: GAPDH (Hs99999905_m1), CD3 (Hs00167894_m1), FoxP3 (Hs01085834_m1), PD-L1 (Hs01125301_m1), PD-L2 (Hs00228839_m1), GRAIL (Hs00226053_m1), CBL-B (Hs00180288_ $\mathrm{m} 1$ ), and ITCH (Hs00395201_m1).

shRNA knockdown of $m$ TOR, raptor, and rictor. MTOR, raptor, or rictor was silenced in ECs using lentivirus-mediated transduction of shRNA. To generate lentivirus, plasmids encoding mTOR shRNA (mTOR_2 shRNA), raptor shRNA (raptor_2 shRNA), rictor shRNA (rictor_1 shRNA), or control GFP shRNA (pLKO.1 GFP shRNA) were transfected into 293T cells together with the CMV-dR8.2 packaging and CMV-VSVG envelope plasmids, using Lipofectamine 2000 (Invitrogen) according to the manufacturer's instructions. Virus containing supernatants was collected at 48 and 72 hours 
after transfection and used to infect ECs in the presence of $8 \mu \mathrm{g} / \mathrm{ml}$ Polybrene. Following two rounds of infection, transduced cells were selected via puromycin resistance and used for experiments 5 days after infection. Knockdown was confirmed with Western blot analysis. mTOR_2 shRNA (85) (Addgene plasmid 1856), rictor_1 shRNA (85) (Addgene plasmid 1853), raptor_2 shRNA (85) (Addgene plasmid 1858), and pLKO.1 GFP shRNA (Addgene plasmid 30323) were deposited by David Sabatini (Massachusetts Institute of Technology, Cambridge, Massachusetts, USA); CMV-dR8.2 and CMV-VSVG (Addgene plasmid 8455 and 8454) were deposited by Robert Weinberg (Massachusetts Institute of Technology).

FoxP3 locus methylation analysis. FoxP3 locus methylation analysis was performed as previously described (27). Briefly, human $\mathrm{CD}^{+}$memory $\mathrm{T}$ cells stimulated by rapa-ECs were stained with fluorescent antibodies and sorted on the basis of high expression of CD25 and low/negative expression of CD127. Natural Tregs were sorted (based on the same markers) from unstimulated $\mathrm{CD}^{+} \mathrm{T}$ cells and served as positive control. Genomic DNA was isolated from sorted $\mathrm{CD} 44^{+} \mathrm{CD} 25^{\text {hi }} \mathrm{CD} 127^{\text {lo }} \mathrm{T}$ cells using DNeasy Blood and Tissue Kit (QIAGEN). Sodium bisulfite treatment was performed on purified genomic DNA using EpiTect Bisulfite Kit (QIAGEN). Bisulfite-treated DNA was PCR amplified using the following primers: p-5'TGTTTGGGGGTAGAGGATTT-3' and o-5'-TATCACCCCACCTAAACCAA- $3^{\prime}$. Amplified DNA product was gel purified and sequenced directly. Trace files were interpreted using 4Peaks software (Mekentosj).

Immunoblotting. ECs cultured in 6-well plates were washed with ice-cold PBS and lysed directly by addition of NP40 lysis buffer $(50 \mathrm{mM}$ Tris- $\mathrm{HCl}$, $10 \%$ glycerol, $125 \mathrm{mM} \mathrm{NaCl}, 1 \%$ Nonidet P-40, $5.3 \mathrm{mM} \mathrm{NaF}, 1 \mathrm{mM}$ orthovanadate, and $1 \mathrm{mg} / \mathrm{ml}$ protease inhibitor mixture [Roche]). EC lysates were frozen, thawed on ice, and cleared by centrifugation at 12,000 $\mathrm{g}$ for $10 \mathrm{~min}$ utes. The protein concentration of each lysate was determined using DC Protein Assay (Bio-Rad), and equal amounts of protein from each sample were boiled at $95^{\circ} \mathrm{C}$ in SDS sample buffer for 10 minutes. Samples were separated by electrophoresis in a $10 \%$ SDS-PAGE gel, and proteins were transferred to PVDF membrane (Millipore) at $100 \mathrm{~V}$ for 1 hour at $4^{\circ} \mathrm{C}$. Following blocking in TBST containing 5\% BSA, membranes were incubated overnight at $4{ }^{\circ} \mathrm{C}$ with anti-mTOR, anti-rictor, anti-raptor, anti-phospho-S6K1, or anti-actin antibodies (all from Cell Signaling Technology). Bound antibodies were detected with HRP-conjugated goat anti-mouse or goat anti-rabbit secondary antibodies (Jackson ImmunoResearch Laboratories Inc.) and SuperSignal West Pico Chemiluminescent Substrate (Pierce).

ELISA. Supernatants from EC-T cell cocultures were collected after 24 hours of stimulation and analyzed by ELISA (eBioscience) for IL-2 and IFN- $\gamma$, according to the manufacturer's instructions. For IL- 6 experiments, ECs were treated with rapamycin $(10 \mathrm{ng} / \mathrm{ml})$ for 2 hours and then treated with $50 \mathrm{ng} / \mathrm{ml} \mathrm{IFN-} \gamma$ or $1 \mathrm{ng} / \mathrm{ml} \mathrm{TNF-} \alpha$ (R\&D), in the continued presence of rapamycin. Supernatant was collected after 24 hours and analyzed by ELISA (eBioscience) for IL-6.

In vivo rapamycin treatment. Rapamycin (Sirolimus, Calbiochem) was diluted in ethanol and mixed with an equal volume of Cremophor EL (Sigma-Aldrich). This mixture was dissolved in Dulbecco's PBS at a final volume of $2 \mathrm{ml} / \mathrm{mouse}$ and administered via intraperitoneal injection. Mice were injected with $3 \mathrm{mg} / \mathrm{kg}$ sirolimus every 24 hours for 3 days. Control mice were injected with a mixture containing ethanol and Cremophor EL in PBS.

Arterial transplantation. Arterial transplantation was performed as previously described (53). Briefly, 1- to 3-mm segments of human artery were interposed into the infrarenal aortae of female C.B-17 SCID/beige mice (Taconic) by end-to-end anastomosis and left to heal for 30 days. Adjacent segments of human artery were transplanted into $2-5$ mice for each experiment, and data from individual experiments were pooled for analysis. After 30 days, transplanted mice with healed-in grafts were treated with vehicle or rapamycin (as described above) for 72 hours. Following treatment, mice were anesthetized, and the treated human arterial grafts, along with a 1- to 2-mm cuff of mouse aorta on each end, were harvested and re-transplanted into a second SCID/beige mouse previously reconstituted with PBMCs allogeneic to the arterial graft. After 10-21 days, these secondary recipients were anesthetized, and arterial grafts were perfused with normal saline and excised before death. Grafts were snap frozen in optimal cutting temperature (OCT) compound (Sakura Finetek). Serial 5 - $\mu \mathrm{m}$ transverse sections were cut for morphometric and immunofluorescence analysis.

For adoptive transfer of human $\mathrm{T}$ cells, SCID/beige mice were given an intraperitoneal injection of $3 \times 10^{8}$ human PBMCs (allogeneic to the artery graft) from a healthy adult volunteer donor 10-14 days prior to artery implantation. As previously described, only $\mathrm{T}$ and $\mathrm{B}$ lymphocytes are successfully engrafted by this procedure, and only $\mathrm{T}$ cells appear in the circulation $(53,55)$. Engraftment was assessed 7 days after PBMC administration via flow cytometry analysis of heparinized blood, collected by retro-orbital puncture, stained with FITC-conjugated anti-human CD3 (Beckman Coulter) and PE-conjugated anti-mouse CD45 (BD Biosciences). Engraftment was considered successful when a distinct population of human $\mathrm{CD}^{+}$cells (typically ranging from $0.5 \%$ to $10 \%$ of the total mononuclear cell population) was observed. In PD-1 blockade experiments, we observed that injection of intact neutralizing PD-1 antibodies (BioXCell, clone J116) depleted mice of circulating human T cells. To circumvent this problem, we prepared $\mathrm{F}\left(\mathrm{ab}^{\prime}\right)_{2}$ fragments from this antibody and confirmed that these fragments were functional in vitro in cocultures of $\mathrm{CD} 4^{+}$memory $\mathrm{T}$ cells with rapa-ECs and no longer depleted $\mathrm{T}$ cells in vivo. $\mathrm{F}\left(\mathrm{ab}^{\prime}\right)_{2}$ fragments were prepared using a $\mathrm{F}\left(\mathrm{ab}^{\prime}\right)_{2}$ preparation kit (Thermo Scientific) according to the manufacturer's instructions. For PD-1 blockade, mice with rapamycin-pretreated grafts were injected subcutaneously with a neutralizing PD-1 $\mathrm{F}\left(\mathrm{ab}^{\prime}\right)_{2}$ fragment or an irrelevant $\operatorname{IgG} 1 \mathrm{~F}\left(\mathrm{ab}^{\prime}\right)_{2}$ fragment (BioXCell, MOPC-21), starting with a 200- $\mu$ g loading dose the day before re-transplant and then a subsequent dose of $100 \mu \mathrm{g} 3$ times per week. Grafts were harvested 14 days after re-transplant.

Histology and immunofluorescence microscopy. Five-micrometer cross sections of artery grafts were stained with Elastica-van Gieson (EVG) and used for morphometric analysis. Total vessel area (determined as the area bound by the external elastic lamina and the lumen), mean intimal area (determined as the area bound by the internal elastic lamina and the lumen), and luminal area were quantified using ImageJ software (NIH).

For immunofluorescence microscopy, serial sections were stained with purified mouse anti-human PD-L1 and PD-L2 antibodies (eBioscience) and an Alexa Fluor 546-conjugated donkey anti-mouse secondary antibody (Invitrogen). ECs were stained with biotinylated Ulex europaeus agglutinin I (Ulex, Vector Laboratories) and Alexa Fluor 488-conjugated avidin (Invitrogen). Immunofluorescence intensity of PD-L1 and PD-L2 staining was quantified using ImageJ by first highlighting Ulex-positive cells and then determining the mean pixel intensity, in the red channel, on highlighted cells. All fluorescence intensity values were normalized to those obtained from vehicle-treated grafts. Graft-infiltrating $\mathrm{T}$ cells were stained with purified rabbit anti-human CD3 (Dako) and an Alexa Fluor 546-conjugated donkey anti-rabbit secondary antibody (Invitrogen). Immunofluorescence images were acquired with an Axiovert 200M fluorescence microscope (Zeiss) equipped with an ORCA-AG digital camera (Hamamatsu) using Volocity software (PerkinElmer). An FITC filter was used to detect Alexa Fluor 488-stained cells, and a tetramethylrhodamine isothiocyanate filter was used to detect Alexa Fluor 594-stained cells. Total numbers of intimal $\mathrm{CD}^{+} \mathrm{T}$ cells were determined in 3 cross sections per graft.

Statistics. All data are expressed as mean \pm SEM. Statistical comparisons were made using Student's $t$ test with the Bonferroni correction as appropriate. In all experiments, unless otherwise stated, $P$ values of 0.05 or less were considered statistically significant. 
Study approval. All human cells were obtained under protocols approved by the Institutional Review Board of Yale University. Healthy adult blood donors gave informed consent. All experimental animal protocols were approved by the Yale University Institutional Animal Care and Use Committee.

\section{Acknowledgments}

We thank L. Benson and G. Davis for expert assistance in EC culture and animal care. This work is supported by NIH grants P01HL070295 to J.S. Pober and G. Tellides, R01-HL109455 to J.S. Pober, and AI06925 and AI095261 to U.H. von Andrian. C. Wang was supported by an NIH Medical Scientist Training Program grant (T32-GM007205) and is currently supported by an NIH National Research Service Award (NRSA) predoctoral fellowship (F30HL114253).

Received for publication August 6, 2012, and accepted in revised form January 17, 2013.

Address correspondence to: Jordan S. Pober, Yale University School of Medicine, 10 Amistad St., Room 401D, New Haven, Connecticut 06520-8089, USA. Phone: 203.737.2292; Fax: 203.737.2293; E-mail: jordan.pober@yale.edu.
1. Issa F, Schiopu A, Wood KJ. Role of T cells in graft rejection and transplantation tolerance. Expert Rev Clin Immunol. 2010;6(1):155-169.

2. Pober JS, Ma W, Biedermann B, Libby P. Vascular cells have limited capacities to activate and differentiate T cells: implications for transplant vascular sclerosis. Transpl Immunol. 1997;5(4):251-254.

3. Salomon RN, Hughes CC, Schoen FJ, Payne DD, Pober JS, Libby P. Human coronary transplantation-associated arteriosclerosis. Evidence for a chronic immune reaction to activated graft endothelial cells. Am J Pathol. 1991;138(4):791-798.

4. Shiao SL, McNiff JM, Pober JS. Memory T cells and their costimulators in human allograft injury. J Immunol. 2005;175(8):4886-4896.

5. Fogal B, et al. Neutralizing IL-6 reduces human arterial allograft rejection by allowing emergence of CD161+CD4+ regulatory T cells. J Immunol. 2011;187(12):6268-6280.

6. Rao DA, Tracey KJ, Pober JS. IL-1alpha and IL-1beta are endogenous mediators linking cell injury to the adaptive alloimmune response. J Immunol. 2007;179(10):6536-6546.

7. Oppenheimer-Marks N, Brezinschek RI, Mohamadzadeh M, Vita R, Lipsky PE. Interleukin 15 is produced by endothelial cells and increases the transendothelial migration of $\mathrm{T}$ cells In vitro and in the SCID mouse-human rheumatoid arthritis model In vivo. J Clin Invest. 1998;101(6):1261-1272.

8. Yamaoka-Tojo M, et al. C-reactive protein-induced production of interleukin-18 in human endothelial cells: a mechanism of orchestrating cytokine cascade in acute coronary syndrome. Heart Vessels. 2003;18(4):183-187.

9. Dengler TJ, Pober JS. Human vascular endothelial cells stimulate memory but not naive CD8+ T cells to differentiate into CTL retaining an early activation phenotype. J Immunol. 2000;164(10):5146-5155.

10. Shiao SL, Kirkiles-Smith NC, Shepherd BR, McNiff JM, Carr EJ, Pober JS. Human effector memory CD4+ $\mathrm{T}$ cells directly recognize allogeneic endothelial cells in vitro and in vivo. J Immunol. 2007;179(7):4397-4404.

11. Choi J, Enis DR, Koh KP, Shiao SL, Pober JS. T lymphocyte-endothelial cell interactions. Annu Rev Immunol. 2004;22:683-709.

12. Rodig N, et al. Endothelial expression of PD-L1 and PD-L2 down-regulates CD8+ T cell activation and cytolysis. Eur I Immunol. 2003;33(11):3117-3126.

13. LaGier AJ, Pober JS. Immune accessory functions of human endothelial cells are modulated by overexpression of B7-H1 (PDL1). Hum Immunol. 2006;67(8):568-578.

14. Lombardi G, Sidhu S, Daly M, Batchelor JR, Makgoba W, Lechler RI. Are primary alloresponses truly primary? Int Immunol. 1990;2(1):9-13.

15. Valujskikh A, Lakkis FG. In remembrance of things past: memory $\mathrm{T}$ cells and transplant rejection. Immunol Rev. 2003;196:65-74.

16. Adams AB, et al. Heterologous immunity provides a potent barrier to transplantation tolerance. J Clin Invest. 2003;111(12):1887-1895.

17. Heeger PS, et al. Pretransplant frequency of donor-specific, IFN-gamma-producing lymphocytes is a manifestation of immunologic memory and correlates with the risk of posttransplant rejection episodes. J Immunol. 1999;163(4):2267-2275.

18. Pober JS, Tellides G. Participation of blood vessel cells in human adaptive immune responses. Trends Immunol. 2012;33(1):49-57.

19. Lechler RI, Batchelor JR. Restoration of immunogenicity to passenger cell-depleted kidney allografts by the addition of donor strain dendritic cells. J Exp Med. 1982;155(1):31-41.

20. Zhu J, Yamane H, Paul WE. Differentiation of effector CD4 T cell populations (*). Annu Rev Immunol. 2010;28:445-489.

21. O'Shea JJ, Paul WE. Mechanisms underlying lineage commitment and plasticity of helper CD4+ T cells. Science. 2010;327(5969):1098-1102.

22. Le Moine A, Goldman M, Abramowicz D. Multiple pathways to allograft rejection. Transplantation. 2002;73(9):1373-1381.

23. Atalar K, Afzali B, Lord G, Lombardi G. Relative roles of Th1 and Th17 effector cells in allograft rejection. Curr Opin Organ Transplant. 2009; 14(1):23-29.

24. Benghiat FS, Charbonnier LM, Vokaer B, De Wilde $\mathrm{V}$, Le Moine A. Interleukin 17-producing T helper cells in alloimmunity. Transplant Rev (Orlando). 2009;23(1):11-18.

25. Afzali B, Lombardi G, Lechler RI, Lord GM. The role of T helper 17 (Th17) and regulatory T cells (Treg) in human organ transplantation and autoimmune disease. Clin Exp Immunol. 2007;148(1):32-46.

26. Curotto de Lafaille MA, Lafaille JJ. Natural and adaptive foxp $3+$ regulatory $\mathrm{T}$ cells: more of the same or a division of labor? Immunity. 2009;30(5):626-635.

27. Floess $S$, et al. Epigenetic control of the foxp 3 locus in regulatory T cells. PLoS Biol. 2007;5(2):e38.

28. Sakaguchi S, Miyara M, Costantino CM, Hafler DA. FOXP3 + regulatory $\mathrm{T}$ cells in the human immune system. Nat Rev Immunol. 2010;10(7):490-500.

29. Cobbold SP, et al. Induction of foxP3 + regulatory $\mathrm{T}$ cells in the periphery of $\mathrm{T}$ cell receptor transgenic mice tolerized to transplants. J Immunol. 2004;172(10):6003-6010.

30. Kretschmer K, Apostolou I, Hawiger D, Khazaie K, Nussenzweig MC, von Boehmer H. Inducing and expanding regulatory $\mathrm{T}$ cell populations by foreign antigen. Nat Immunol. 2005;6(12):1219-1227.

31. Nadig SN, et al. In vivo prevention of transplant arteriosclerosis by ex vivo-expanded human regulatory T cells. Nat Med. 2010;16(7):809-813.

32. Kingsley CI, Karim M, Bushell AR, Wood KJ. CD25+CD4+ regulatory $\mathrm{T}$ cells prevent graft rejection: CTLA-4- and IL-10-dependent immunoregulation of alloresponses. J Immunol. 2002;168(3):1080-1086

33. You S, Leforban B, Garcia C, Bach JF, Bluestone JA, Chatenoud L. Adaptive TGF-beta-dependent regulatory $\mathrm{T}$ cells control autoimmune diabetes and are a privileged target of anti-CD3 antibody treatment. Proc Natl Acad Sci U S A. 2007;104(15):6335-6340.

34. Levings MK, Sangregorio R, Galbiati F, Squadrone
S, de Waal Malefyt R, Roncarolo MG. IFN-alpha and IL-10 induce the differentiation of human type $1 \mathrm{~T}$ regulatory cells. J Immunol. 2001;166(9):5530-5539.

35. Maldonado RA, von Andrian UH. How tolerogenic dendritic cells induce regulatory T cells. Adv Immunol. 2010;108:111-165.

36. Francisco LM, et al. PD-L1 regulates the development, maintenance, and function of induced regulatory T cells. J Exp Med. 2009;206(13):3015-3029.

37. Messi M, Giacchetto I, Nagata K, Lanzavecchia A, Natoli G, Sallusto F. Memory and flexibility of cytokine gene expression as separable properties of human $\mathrm{T}(\mathrm{H}) 1$ and $\mathrm{T}(\mathrm{H}) 2$ lymphocytes. Nat Immunol. 2003;4(1):78-86.

38. Lees JR, Farber DL. Generation, persistence and plasticity of CD4 T-cell memories. Immunology. 2010;130(4):463-470.

39. Vukmanovic-Stejic M, et al. The kinetics of CD4+Foxp3+ T cell accumulation during a human cutaneous antigen-specific memory response in vivo. J Clin Invest. 2008;118(11):3639-3650.

40. Saunders RN, Metcalfe MS, Nicholson ML. Rapamycin in transplantation: a review of the evidence. Kidney Int. 2001;59(1):3-16.

41. Sengupta S, Peterson TR, Sabatini DM. Regulation of the mTOR complex 1 pathway by nutrients, growth factors, and stress. Mol Cell. 2010;40(2):310-322.

42. Sarbassov DD, et al. Prolonged rapamycin treatment inhibits mTORC 2 assembly and Akt/PKB. Mol Cell. 2006;22(2):159-168.

43. Powell JD, Pollizzi KN, Heikamp EB, Horton MR. Regulation of Immune Responses by mTOR. Annu Rev Immunol. 2012;30:39-68.

44. Hackstein H, et al. Rapamycin inhibits IL-4--induced dendritic cell maturation in vitro and dendritic cell mobilization and function in vivo. Blood. 2003;101(11):4457-4463.

45. Taner T, Hackstein H, Wang Z, Morelli AE, Thomson AW. Rapamycin-treated, alloantigen-pulsed host dendritic cells induce ag-specific T cell regulation and prolong graft survival. Am J Transplant. 2005;5(2):228-236.

46. Turnquist HR, Raimondi G, Zahorchak AF, Fischer RT, Wang Z, Thomson AW. Rapamycin-conditioned dendritic cells are poor stimulators of allogeneic CD4+ T cells, but enrich for antigen-specific Foxp3 $+\mathrm{T}$ regulatory cells and promote organ transplant tolerance. J Immunol. 2007; 178(11):7018-7031.

47. Horibe EK, et al. Rapamycin-conditioned, alloantigen-pulsed dendritic cells promote indefinite survival of vascularized skin allografts in association with $\mathrm{T}$ regulatory cell expansion. Transpl Immunol. 2008;18(4):307-318

48. Wang J, Ioan-Facsinay A, van der Voort EI, Huizinga TW, Toes RE. Transient expression of FOXP3 in human activated nonregulatory CD4+ T cells. Eur J Immunol. 2007;37(1):129-138.

49. Allan SE, et al. Activation-induced FOXP3 in human $\mathrm{T}$ effector cells does not suppress proliferation or cytokine production. Int Immunol. 2007;19(4):345-354.

50. Manes TD, Shiao SL, Dengler TJ, Pober JS. TCR sig- 
naling antagonizes rapid IP-10-mediated transendothelial migration of effector memory CD4+ T cells. J Immunol. 2007;178(5):3237-3243.

51. Keir ME, Butte MJ, Freeman GJ, Sharpe AH. PD-1 and its ligands in tolerance and immunity. Annu Rev Immunol. 2008;26:677-704.

52. Fujimoto $M$, et al. The influence of excessive IL- 6 production in vivo on the development and function of Foxp3+ regulatory T cells. J Immunol. 2011;186(1):32-40

53. Yi T, et al. Reperfusion injury intensifies the adaptive human $\mathrm{T}$ cell alloresponse in a human-mouse chimeric artery model. Arterioscler Thromb Vasc Biol. 2012;32(2):353-360.

54. Tellides $\mathrm{G}$, et al. Interferon-gamma elicits arteriosclerosis in the absence of leukocytes. Nature. 2000;403(6766):207-211.

55. Pober JS, Bothwell AL, Lorber MI, McNiff JM, Schechner JS, Tellides G. Immunopathology of human $\mathrm{T}$ cell responses to skin, artery and endothelial cell grafts in the human peripheral blood lymphocyte/severe combined immunodeficient mouse. Springer Semin Immunopathol. 2003;25(2):167-180.

56. Krupnick AS, et al. Murine vascular endothelium activates and induces the generation of allogeneic CD4+25+Foxp3+ regulatory T cells. J Immunol. 2005;175(10):6265-6270.

57. Tellides G, Pober JS. Interferon-gamma axis in graft arteriosclerosis. Circ Res. 2007;100(5):622-632.

58. van Loosdregt J, et al. The chemokine and chemokine receptor profile of infiltrating cells in the wall of arteries with cardiac allograft vasculopathy is indicative of a memory T-helper 1 response. Circulation. 2006;114(15):1599-1607.

59. Araki K, Ellebedy AH, Ahmed R. TOR in the immune system. Curr Opin Cell Biol. 2011; 23(6):707-715.

60. Keir ME, et al. Tissue expression of PD-L1 mediates peripheral T cell tolerance. J Exp Med. 2006;203(4):883-895

61. Parry RV, et al. CTLA-4 and PD-1 receptors inhibit
T-cell activation by distinct mechanisms. Mol Cell Biol. 2005;25(21):9543-9553.

62. Ito $\mathrm{T}$, et al. Analysis of the role of negative $\mathrm{T}$ cell costimulatory pathways in CD4 and CD8 T cellmediated alloimmune responses in vivo. J Immunol. 2005;174(11):6648-6656.

63. Hori J, et al. B7-H1-induced apoptosis as a mechanism of immune privilege of corneal allografts. $J$ Immunol. 2006;177(9):5928-5935.

64 . Sandner SE, et al. Role of the programmed death-1 pathway in regulation of alloimmune responses in vivo. J Immunol. 2005;174(6):3408-3415.

65. Ozkaynak E, et al. Programmed death-1 targeting can promote allograft survival. J Immunol. 2002;169(11):6546-6553

66. Kishimoto T. Interleukin-6: from basic science to medicine -40 years in immunology. Annu Rev Immunol. 2005;23:1-21.

67. Dienz O, Rincon M. The effects of IL- 6 on CD4 T cell responses. Clin Immunol. 2009;130(1):27-33.

68. McLoughlin RM, et al. IL-6 trans-signaling via STAT3 directs T cell infiltration in acute inflammation. Proc Natl Acad Sci U S A. 2005;102(27):9589-9594.

69. Boratynska M, Klinger M, Szyber P, Patrzalek D, Polak K. Interleukin-6 in chronic renal allograft rejection: influence of nonimmunologic risk factors. Transplant Proc. 2001;33(1-2):1215-1217.

70. Plenz G, et al. The interleukin-6/interleukin-6-receptor system is activated in donor hearts. J Am Coll Cardiol. 2002;39(9):1508-1512.

71. Pasare C, Medzhitov R. Toll pathway-dependent blockade of CD4+CD25+ T cell-mediated suppression by dendritic cells. Science. 2003; 299(5609):1033-1036.

72. Wood KJ, Sakaguchi S. Regulatory T cells in transplantation tolerance. Nat Rev Immunol. 2003;3(3):199-210.

73. Li Y, et al. Analyses of peripheral blood mononuclear cells in operational tolerance after pediatric living donor liver transplantation. Am J Transplant. 2004;4(12):2118-2125.

74. Akl A, et al. An investigation to assess the potential of CD25highCD4+ T cells to regulate responses to donor alloantigens in clinically stable renal transplant recipients. Transpl Int. 2008;21(1):65-73.

75. Weichhart T, et al. The TSC-mTOR signaling pathway regulates the innate inflammatory response. Immunity. 2008;29(4):565-577.

76. Araki K, et al. mTOR regulates memory CD8 T-cell differentiation. Nature. 2009;460(7251):108-112.

77. Zoncu R, Efeyan A, Sabatini DM. mTOR: from growth signal integration to cancer, diabetes and ageing. Nat Rev Mol Cell Biol. 2011;12(1):21-35.

78. Nocentini G, Riccardi C. GITR: a modulator of immune response and inflammation. Adv Exp Med Biol. 2009;647:156-173.

79. Hori J, Taniguchi H, Wang M, Oshima M, Azuma M. GITR ligand-mediated local expansion of regulatory $\mathrm{T}$ cells and immune privilege of corneal allografts. Invest Ophthalmol Vis Sci. 2010;51(12):6556-6565.

80. Ray A, Basu S, Williams CB, Salzman NH, Dittel $\mathrm{BN}$. A novel IL-10-independent regulatory role for $\mathrm{B}$ cells in suppressing autoimmunity by maintenance of regulatory T cells via GITR ligand. J Immunol. 2012;188(7):3188-3198.

81. van Olffen RW, et al. GITR triggering induces expansion of both effector and regulatory CD4+ $\mathrm{T}$ cells in vivo. J Immunol. 2009;182(12):7490-7500.

82. Takeda I, et al. Distinct roles for the OX40-OX 40 ligand interaction in regulatory and nonregulatory T cells. J Immunol. 2004;172(6):3580-3589.

83. Ruby CE, et al. Cutting Edge: OX40 agonists can drive regulatory $\mathrm{T}$ cell expansion if the cytokine milieu is right. J Immunol. 2009;183(8):4853-4857.

84. Walker JD, Maier CL, Pober JS. Cytomegalovirus-infected human endothelial cells can stimulate allogeneic CD4+ memory T cells by releasing antigenic exosomes. J Immunol. 2009; 182(3):1548-1559.

85. Sarbassov DD, Guertin DA, Ali SM, Sabatini DM. Phosphorylation and regulation of Akt/ $\mathrm{PKB}$ by the rictor-mTOR complex. Science. 2005;307(5712):1098-1101. 